THE ASTROPHYSICAL JOURNAL, 336:780-797, 1989 January 15

(C) 1989. The American Astronomical Society. All rights reserved. Printed in U.S.A.

\title{
BLUE STRAGGLER STARS IN THE GLOBULAR CLUSTER NGC 5053
}

\author{
JAMES M. NEMEC \\ Department of Astronomy, University of British Columbia; and Palomar Observatory, California Institute of Technology \\ AND \\ JUDITH G. COHEN \\ Palomar Observatory, California Institute of Technology \\ Received 1988 February 3; accepted 1988 June 28
}

\begin{abstract}
Twenty-four blue straggler stars have been identified in the low central concentration globular cluster NGC 5053. New deep color-magnitude $(C-M)$ diagrams to $23 \mathrm{mag}$, constructed from photometry of over 6000 stars on 4-shooter CCD frames, show that they form a well-defined sequence in the $C$ - $M$ diagram, extending up to $\sim 2.2$ mag brighter than the main-sequence turnoff point. The 12 most luminous blue stragglers are found to be significantly more centrally concentrated than the cluster subgiants with magnitudes in the same interval (a similar result is known for the blue stragglers in the globular cluster NGC 5466). Furthermore, they are also found to be more centrally concentrated than the 12 lower luminosity blue stragglers. Comparisons of the projected radial distributions of the bright and faint blue stragglers, with the radial distributions that are expected for stars of mass $0.8,1.6$, and $2.4 M_{\odot}$ (calculated using multimass King models) suggests that the brightest blue stragglers have an average mass of $\langle M\rangle=1.3 \pm 0.3 M_{\odot}$, which is less than or comparable to twice the mean mass of a main-sequence turnoff star, and the lower luminosity blue stragglers have a mean mass similar to that of the main-sequence turnoff stars (i.e., $M \sim 0.8 M_{\odot}$ ). By fitting theoretical isochrones computed by Bell and VandenBerg to the observed main-sequence turnoff and subgiant branch regions of NGC 5053, a distance modulus of $(m-M)_{0}=16.05 \pm 0.14 \mathrm{mag}$, and an age of $18 \pm 3$ Gyr are derived for NGC 5053. The main-sequence luminosity function shows no sign of "turning over" for stars brighter than $M_{g} \sim 5$ mag.

Subject headings: clusters: globular — stars: binaries — stars: evolution — stars: stellar dynamics
\end{abstract}

\section{INTRODUCTION}

Deep $C$ - $M$ diagrams have now been constructed for several Galactic globular clusters with low central concentrations, such as NGC 288, E3, Pal 5, Pal 12, Pal 14, Pal 15, and NGC 5466 (see Nemec and Harris 1987, hereafter Paper I, for references). Since stars in these clusters have experienced few (if any) two-body encounters over their $\sim 15 \mathrm{Gyr}$ lifetimes (Aarseth and Lecar 1975; Hills and Day 1976), any binary stars in these systems are probably primordial (see Renzini, Mengel, and Sweigart 1977). Candidate binary systems in lowconcentration globular clusters include anomalous Cepheids (Norris and Zinn 1975; Zinn and Searle 1976), CH stars (McClure and Norris 1977; McClure, Fletcher, and Nemec 1980; McClure 1984), and blue stragglers (Hoyle 1964; McCrea 1964; Peterson, Carney, and Latham 1984; Nemec and Harris 1987).

NGC 5053 is one of the most open of all the known globular clusters (core radius $r_{c}=11 \mathrm{pc}=2.5$; concentration $c=$ $\log r_{t} / r_{c}=0.75$, King 1962; Peterson and King 1975), and is notably absent from the above list of clusters. Following its discovery by William Herschel in 1784 , it was not generally recognized as globular cluster until Baade's (1927) identification of nine cluster RR Lyrae stars (see Sawyer 1946; Rosino 1949; Mannino 1963; Sawyer Hogg 1973). Its globular cluster character was further established by the $C$ - $M$ diagram derived by Cuffey (1943), which revealed a redward sloping giant branch. The only modern photometric study of NGC 5053 was made by Sandage, Katem, and Johnson (1977, hereafter SKJ). Their $C$ - $M$ diagram, to $V \sim 17.3 \mathrm{mag}$, defines the principal evolutionary sequences for bright stars in the cluster: a steep red giant branch (RGB) that is extremely blue at the level of the horizontal branch, $(B-V)_{0, g}=0.67 \pm 0.02 \mathrm{mag}$, and a horizontal branch that is populated with RR Lyrae stars and blue horizontal branch stars. The morphology of the $C$ - $M$ diagram is consistent with the cluster's known very low metal abundance (see $\S \mathrm{III} b$ ), and the relatively small number of cluster giants reflects its low luminosity, $M_{V}=-6.1$ mag (Webbink 1985).

In this paper we present a study of NGC 5053 that is based on photometry to $23 \mathrm{mag}$ derived from CCD frames. In $\S$ II the observational data and the photometric methods are described. In $\S$ III deep $C$ - $M$ diagrams are presented, a mean metal abundance for the cluster is derived from the color of the red giant branch at the level of the horizontal branch, and theoretical isochrones are used to derive a distance modulus of $(m-M)_{0}=16.05 \pm 0.14 \mathrm{mag}$ and an age of $18 \pm 3 \mathrm{Gyr}$. A luminosity function based on subgiant and upper mainsequence stars is also constructed. In $\S$ IV, 24 blue stragglers in NGC 5053 are identified and their properties studied. The results are summarized in $\S \mathrm{V}$.

\section{OBSERVATIONS AND PHOTOMETRY}

a) CCD Frames and Data Preprocessing

NGC 5053 was observed through Thuan and Gunn (1976) $g$ and $r$ filters on 1986 February 4/5 with the 4-Shooter CCD camera on the Palomar Observatory 200 inch $(5 \mathrm{~m})$ telescope (see Gunn et al. 1984). A journal of the CCD frames is given in Table 1, and the four fields that were observed are shown in Figure 1 (Plates 19-22). The 4-Shooter is designed around a four-sided reflecting pyramid and four Schmidt cameras mounted at the Cassegrain focus of the Hale telescope. The 


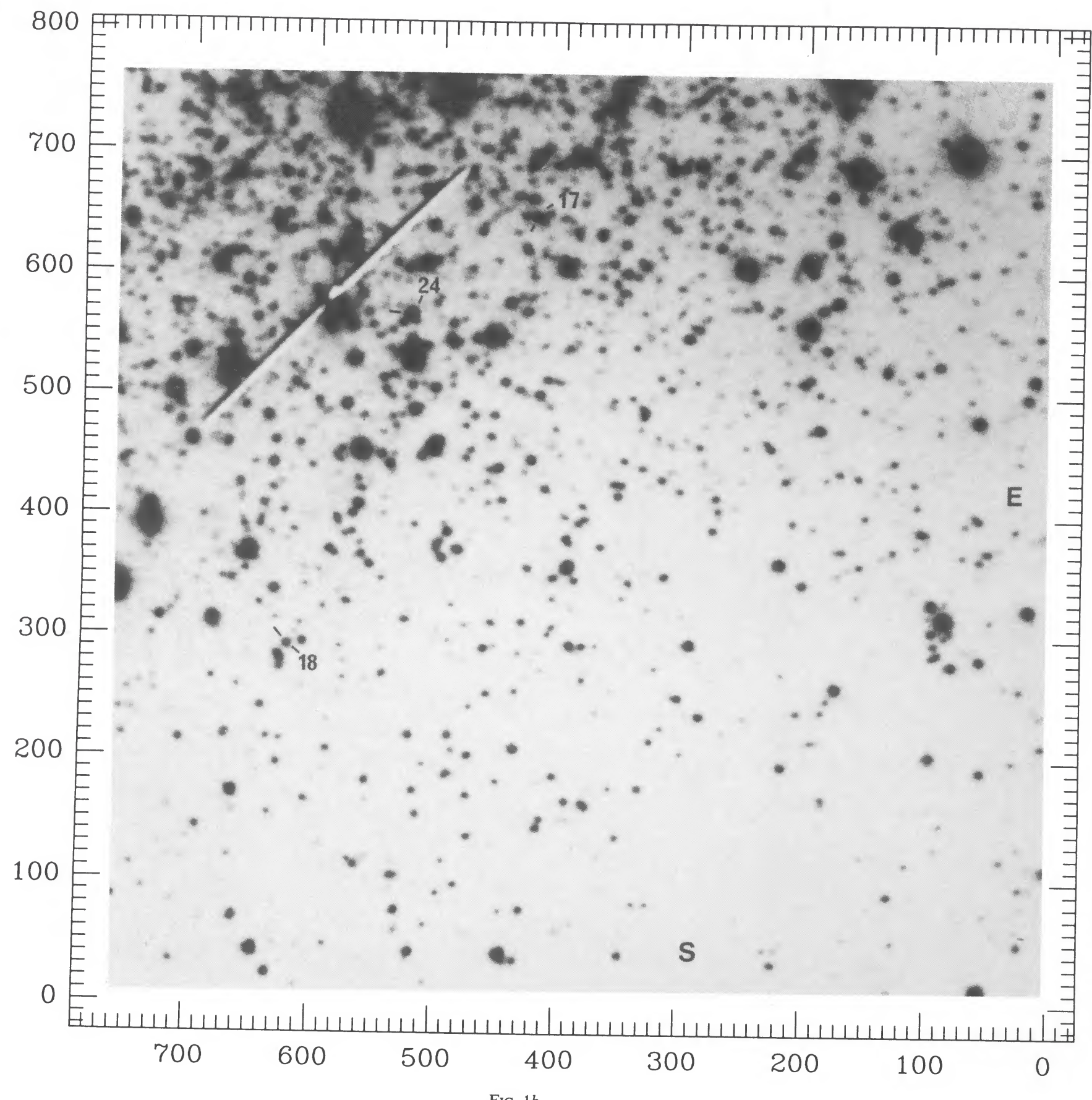

FIG. $1 b$

Nemic AND CoHEN (see 336, 780) 


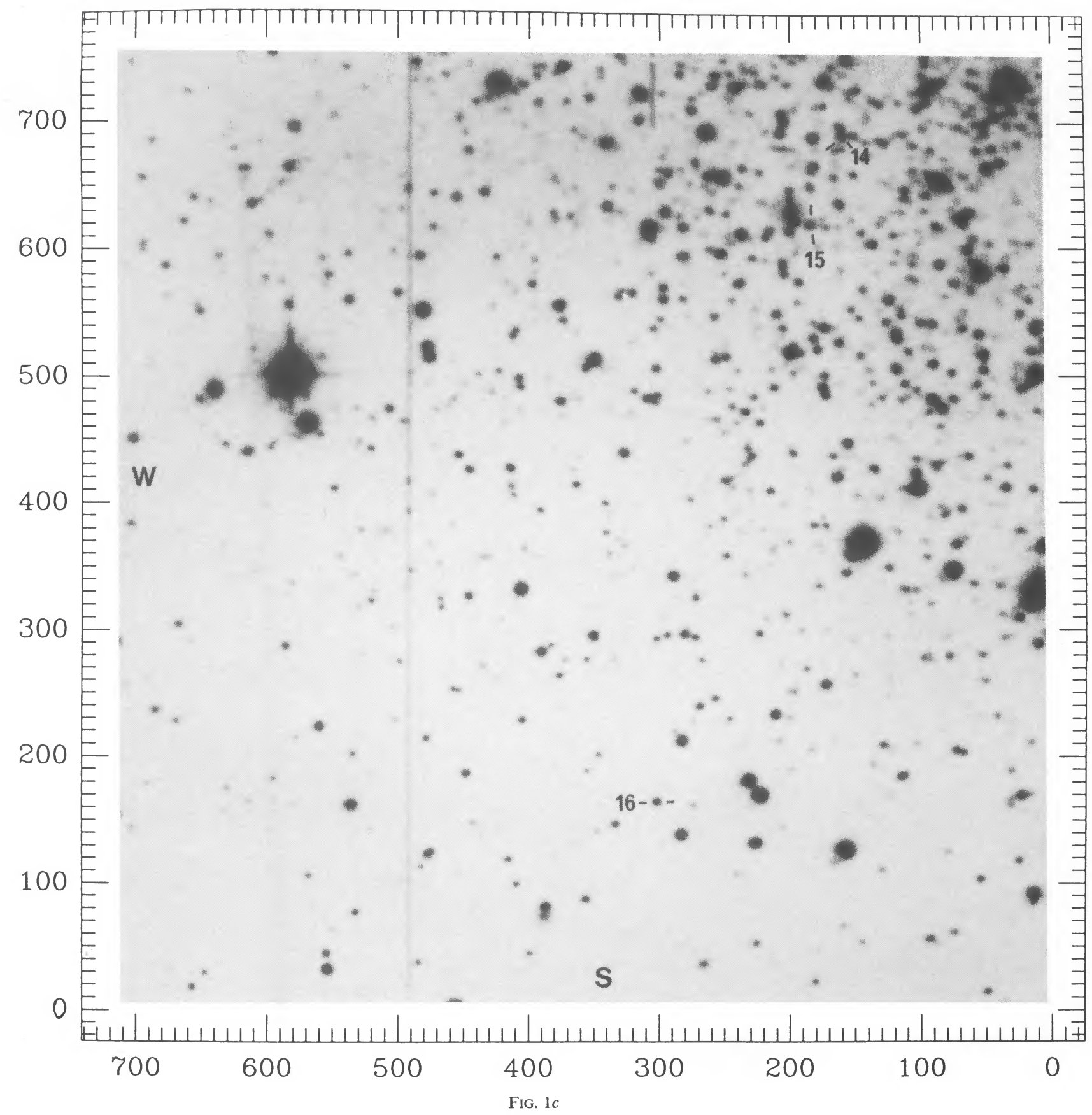

Nemic and Cohen (see 336, 780) 


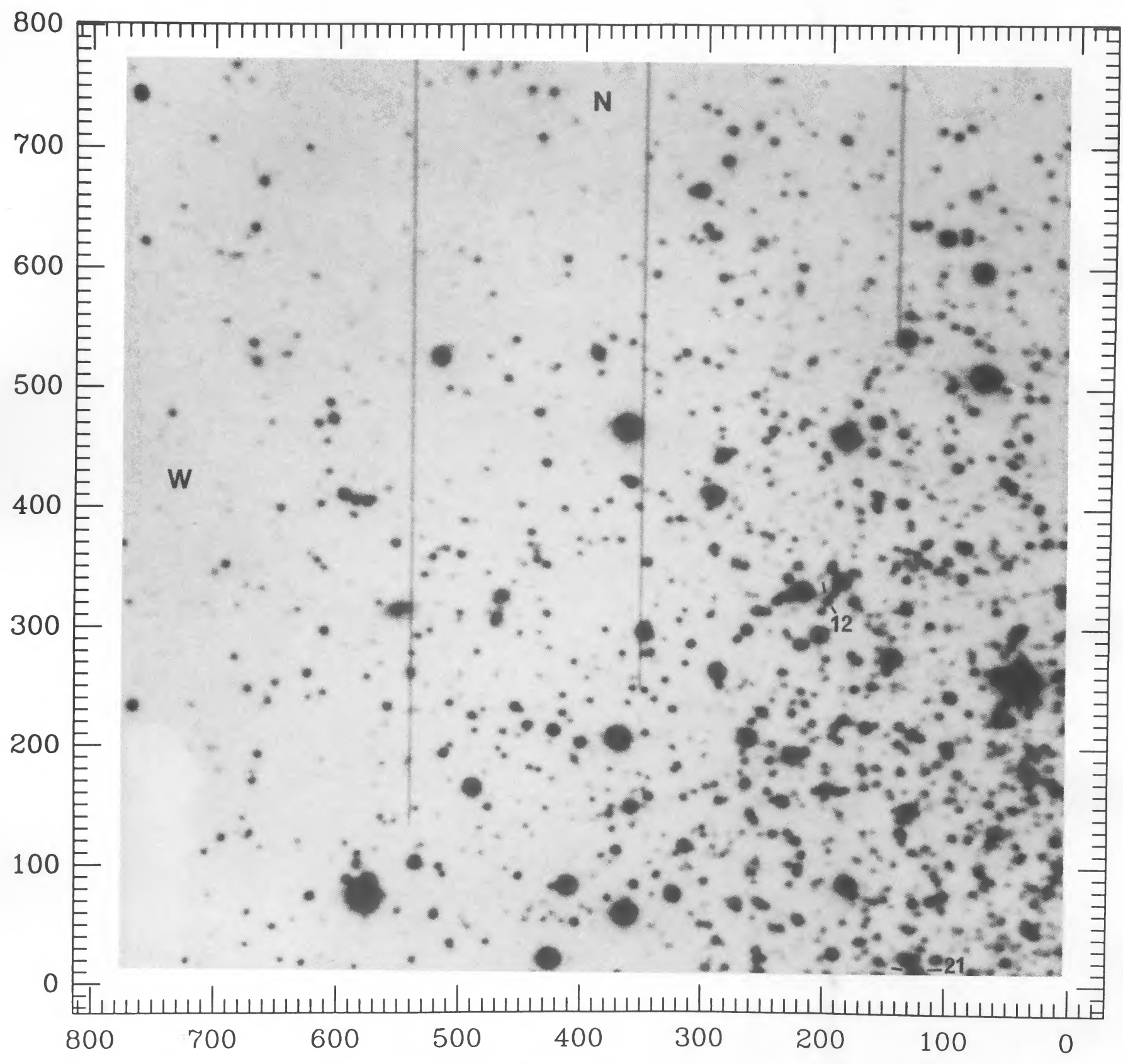

FIG. $1 d$

Nemic AND COHEN (see 336, 780) 


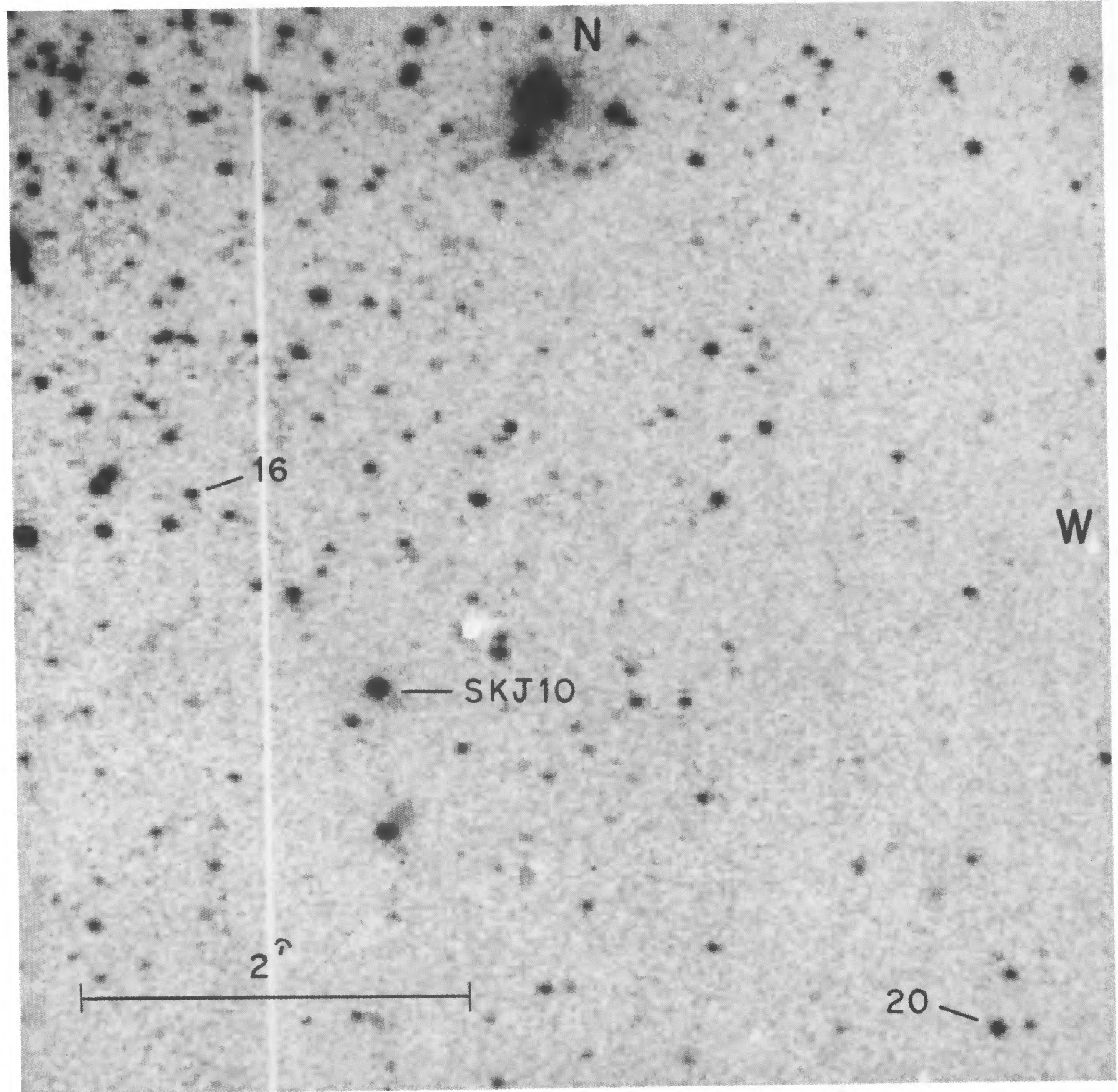

FIG. 7. Identification of blue stragglers 16 and 20. The photograph is from an RCA blue CCD frame ( 320 pixels $\times 512$ pixels) taken with the Palomar 60 inch $(1.5 \mathrm{~m})$ telescope. The two very bright stars at the top of the photograph are SKJ 110 and 111 ; SKJ 10 is also identified.

Nemic AND CoHEN (see 336, 780) 
TABLE 1

CCD OBSERVATIONS OF NGC 5053

\begin{tabular}{|c|c|c|c|c|c|}
\hline Telescope & Filter & $\begin{array}{l}\text { Exposure } \\
\text { (s) }\end{array}$ & U.T. (mid) & FWHM & $\begin{array}{c}\text { Air } \\
\text { Mass }\end{array}$ \\
\hline \multirow[t]{2}{*}{$5.0 \mathrm{~m} \ldots \ldots}$. & $g$ & 400 & 1986 Feb 5, 13:07:58 & $1 . .5$ & 1.07 \\
\hline & $r$ & 300 & 1986 Feb $5,13: 16: 16$ & 1.3 & 1.08 \\
\hline
\end{tabular}

photon detectors in the cameras were Texas Instruments $800 \times 800$ pixel $^{2}$ CCD chips with readout noise $\sim 5$ electrons pixel $^{-1}$, and an image scale size of $0.33 \mathrm{pixel}^{-1}$. The sky was photometric at the time of the observations, and the seeing conditions gave stellar images with FWHM $=1$ ".4. To avoid image saturation at the magnitude level of the blue stragglers the exposure times were deliberately kept short (see Table 1). The imaging of the cluster on the four CCD chips was such that the cluster center (at $\alpha_{1950}=13^{\mathrm{h}} 14^{\mathrm{m}} 0.15, \quad \delta_{1950}^{\mathrm{s}}=$ $+17^{\circ} 57^{\prime} 40^{\prime \prime} .5$, according to Shawl and White 1986) was placed in field 1 near the optical axis of the telescope. On the night of the observations, short out-of-focus exposures of six ThuanGunn standard stars were also taken for calibration purposes.

Before the CCD frames could be used for photometry, bias levels (typically $\sim 300 \mathrm{ADU}_{\text {pixel }}{ }^{-1}$ ) were determined (using the overscan columns) and subtracted, the frames were flattened with debiased dome flats (taken at the end of the night), and edge pixels were trimmed from the original CCD frames. The characteristics of the CCD chips, and a summary of the photometric reductions is given in Table 2.

\section{b) Photometry}

Over 12,000 stellar images were identified on the frames, both automatically and manually. Photometric measurements of the eight frames were made using the aperture and pointspread function (PSF) fitting program DAOPHOT (Stetson 1987). The PSFs for each frame were constructed with 15-20 stars (on average) using the multipass iterative procedure recommended by Stetson. Because of the very low central concentration of NGC 5053, relatively accurate magnitudes and colors were measured right into the cluster center.

A circular aperture with a radius of five pixels (1".7) was used for the aperture photometry, and for the normalization of the final PSF photometry. The local sky measurements were made with an annulus having inner and outer radii of 20 and 25 pixels, respectively. The instrumental 5 pixel magnitudes were transformed to total magnitudes by adding an appropriate correction, which was determined from the growth curves of "cleaned" PSF stars. The growth curve is a plot of the differ- ence between the 5 pixel instrumental magnitude and the $i$ th pixel (where $i=7,9, \ldots 19$ ) instrumental magnitude versus the aperture size. For large apertures, the growth curves level off and the average limiting magnitude difference defines the aperture correction. The mean difference between the magnitude determined using the largest aperture (19 pixel radius) and the second largest aperture (17 pixel radius) was, for every chip, less than $0.006 \mathrm{mag}$. The aperture corrections that were applied to each chip are listed in Table 2.

The instrumental magnitudes (normalized to $1 \mathrm{~s}$ integration time, and corrected to total magnitudes) were transformed to the Thuan-Gunn system using the following equations (established from aperture photometry of the primary standard stars):

$$
\begin{aligned}
(g-r)_{\mathrm{std}} & =-0.020+1.102(g-r)_{\mathrm{instr}}+k_{g-r} X_{g r} \\
r_{\mathrm{std}} & =r_{\mathrm{instr}}+0.098(g-r)_{\mathrm{std}}+k_{r} X_{r},
\end{aligned}
$$

where $X_{r}=$ the airmass at the mid-exposure time of the $r$ exposure, $X_{g r}=$ the average airmass of the $g$ - and $r$-exposures, and the atmospheric extinction coefficients were taken to be $k_{r}=0.10 \mathrm{mag}$ and $k_{g-r}=0.08 \mathrm{mag}$ (well-determined values for Palomar Observatory).

The $g$ and $r$ photometric measurements were paired by star position, using automated procedures, and instrumental $C-M$ diagrams were plotted. Only the photometry of stars with $\mathrm{CHI} \lesssim 4$ (which included almost all of the measurements) was matched, since images with larger $\mathrm{CHI}$ values were deemed to be nonstellar. The following small zero-point shifts in the $g-r$ colors were made to account for the slightly different aperture corrections that were made for each field, and to account for uncertain (but small) zero-point differences: $+0.01 \mathrm{mag}$ for chip $1,-0.03$ mag for chip 3 , and -0.04 mag for chip 4 . These shifts were made so that the main-sequence turnoffs for all the fields were coincident with that of field 2 , the chip on which the Thuan-Gunn standard stars were observed.

Photometry of the $\sim 200$ brightest stars in each of the four fields is given in Table 3, which lists for each star its (internal) star identification number, the $X$ - and $Y$-coordinate, the $g$ magnitude, and the $g-r$ color.

\section{DISCUSSION}

a) Color-Magnitude Diagram to $M_{g} \sim 7$

Calibrated $C-M$ diagrams for the four NGC 5053 fields are presented in Figure 2. The number of stars plotted is 5950, 2571, 1861, and 1517 for Fields $1-4$, respectively. For comparison with other globular cluster $C-M$ diagrams, normal points

TABLE 2

\begin{tabular}{|c|c|c|c|c|c|c|}
\hline $\begin{array}{l}\text { CCD Frame } \\
\text { (1) }\end{array}$ & $\begin{array}{c}\text { Trimmed } \\
\text { Size } \\
\text { (2) }\end{array}$ & $\begin{array}{l}\text { Photons } \\
\text { per ADU } \\
\text { (3) }\end{array}$ & $\begin{array}{c}\text { Readout } \\
\text { Noise } \\
\text { (ADU pixel }^{-1} \text { ) } \\
\text { (4) }\end{array}$ & $\begin{array}{l}\text { Average } \\
\text { Sky } \\
\text { (ADU) } \\
(5)\end{array}$ & $\begin{array}{c}\text { Aperture } \\
\text { Correction } \\
\text { (mag) } \\
(6)\end{array}$ & $\begin{array}{c}\text { Number } \\
\text { of Stars } \\
\text { Measured } \\
\text { (7) }\end{array}$ \\
\hline$g 1(\mathrm{NE})$ & $764 \times 782$ & 2.6 & 4.3 & 1505 & 0.255 & 3371 \\
\hline$g 2(\mathrm{SE})$ & $770 \times 772$ & 2.6 & 4.8 & 1504 & 0.269 & 1148 \\
\hline$g 3(\mathrm{SW})$ & $715 \times 758$ & 2.1 & 4.3 & 1488 & 0.260 & 881 \\
\hline$g 4(\mathrm{NW})$ & $778 \times 774$ & 2.1 & 4.9 & 1512 & 0.242 & 1669 \\
\hline$r 1(\mathrm{NE})$. & $764 \times 782$ & 2.6 & 4.3 & 1894 & 0.223 & 3562 \\
\hline$r$ (SE) & $770 \times 772$ & 2.6 & 4.8 & 1897 & 0.203 & 1251 \\
\hline$r 3(\mathrm{SW})$ & $715 \times 758$ & 2.1 & 4.3 & 1875 & 0.203 & 1193 \\
\hline$r 4(\mathrm{NW})$ & $778 \times 774$ & 2.1 & 4.9 & 1919 & 0.189 & 1455 \\
\hline
\end{tabular}

SUMmaRy OF PHOTOMETRIC RedUCtions 


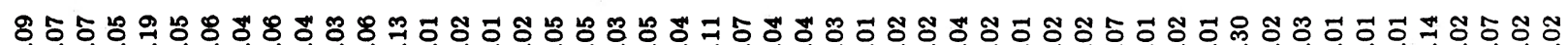

i 000

$\infty$

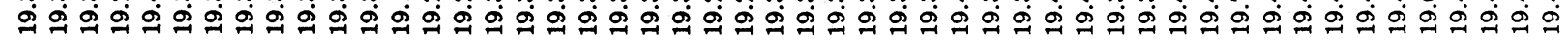

$\succ$

$\times$

บ 0 -

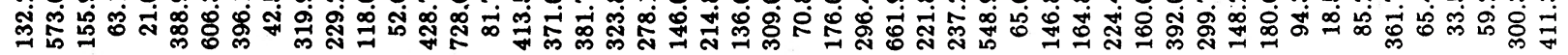

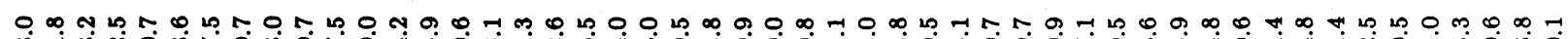

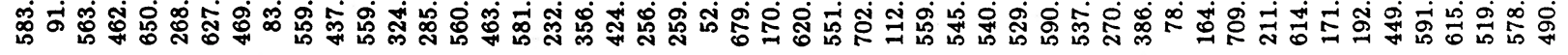

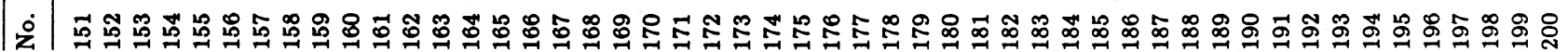

ส

s.

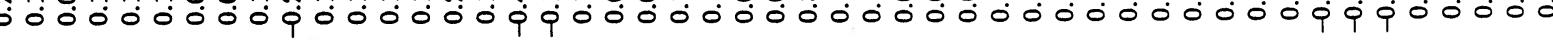

Бు

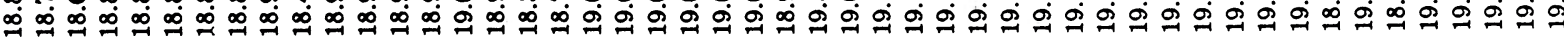

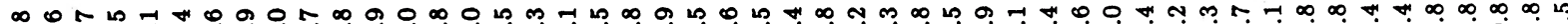

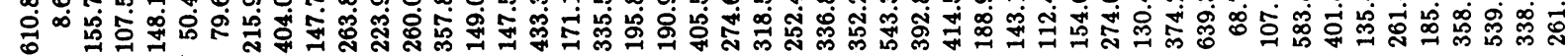

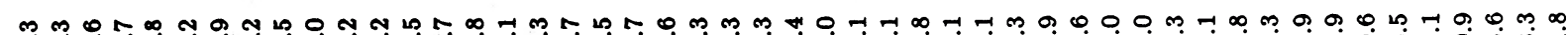

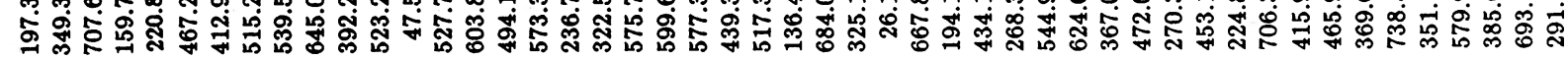

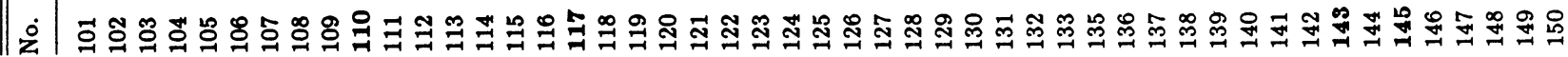

西

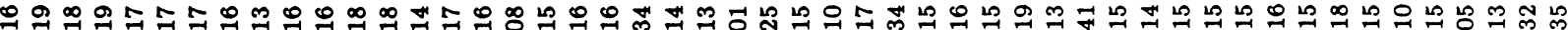

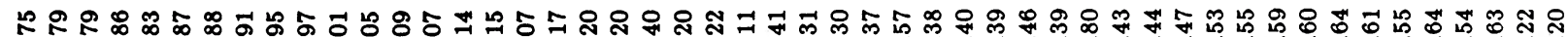

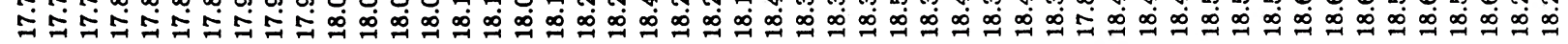

"十

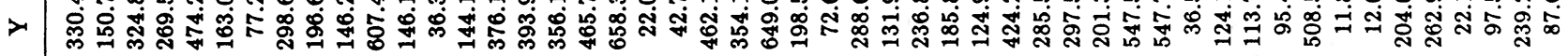

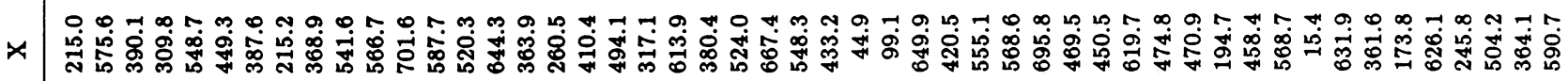
|

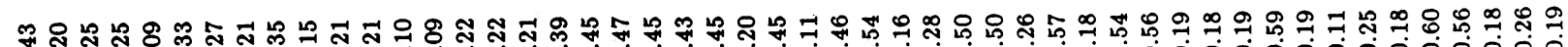

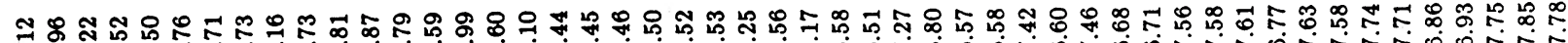

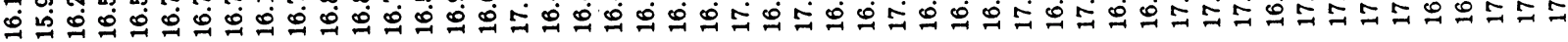

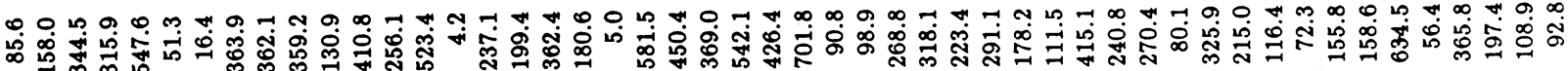
-

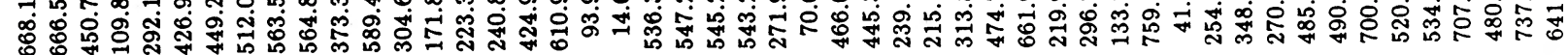

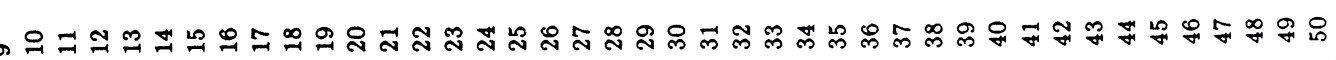




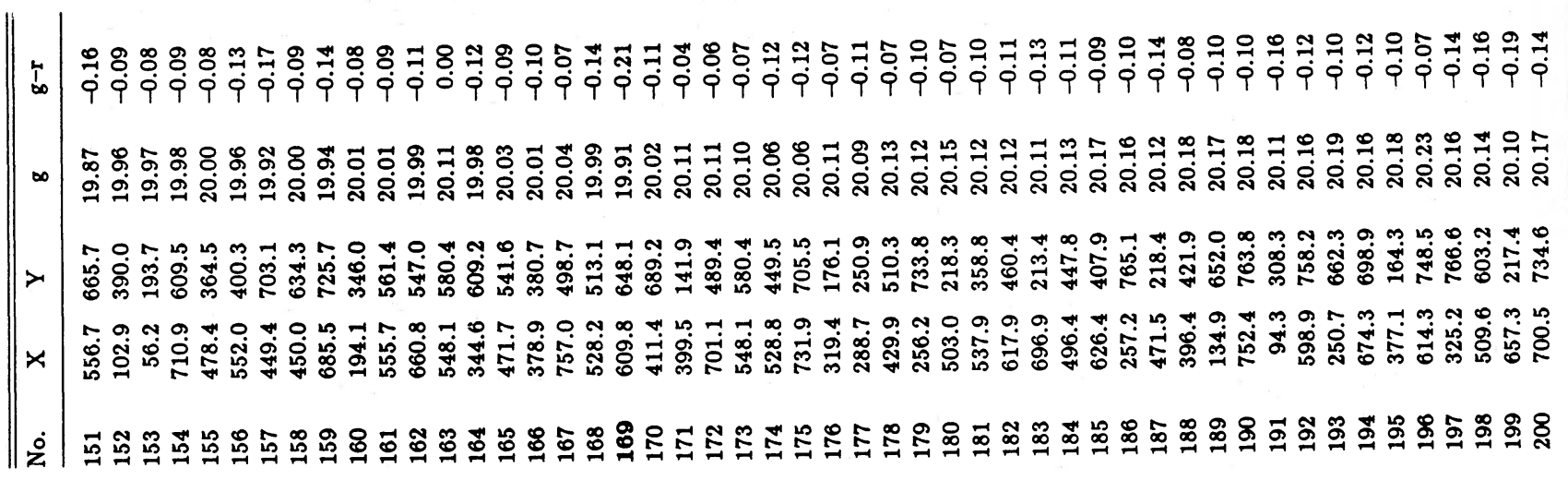

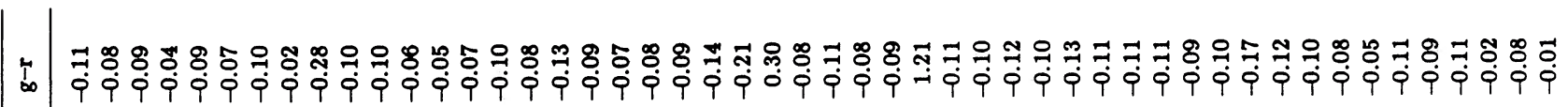

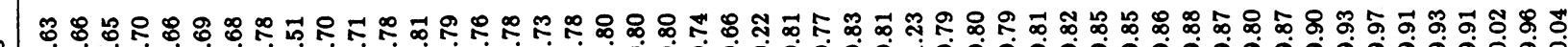
-

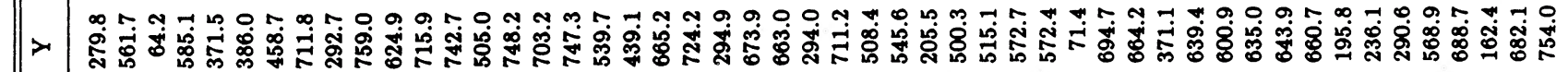
×|

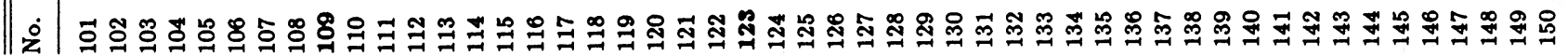

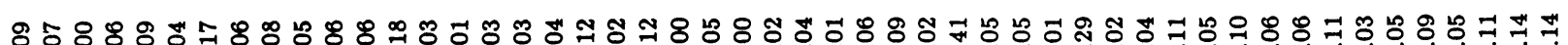
óo.

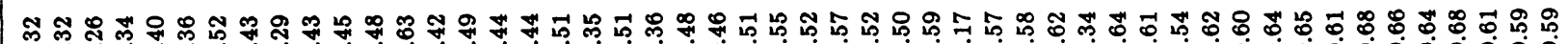

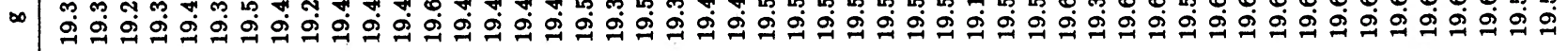

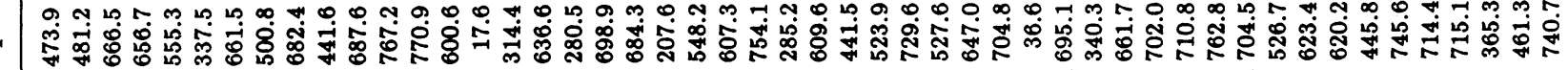
(

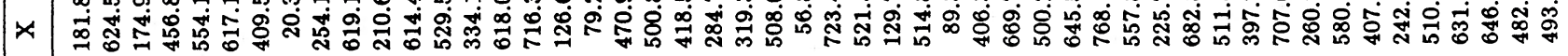
|

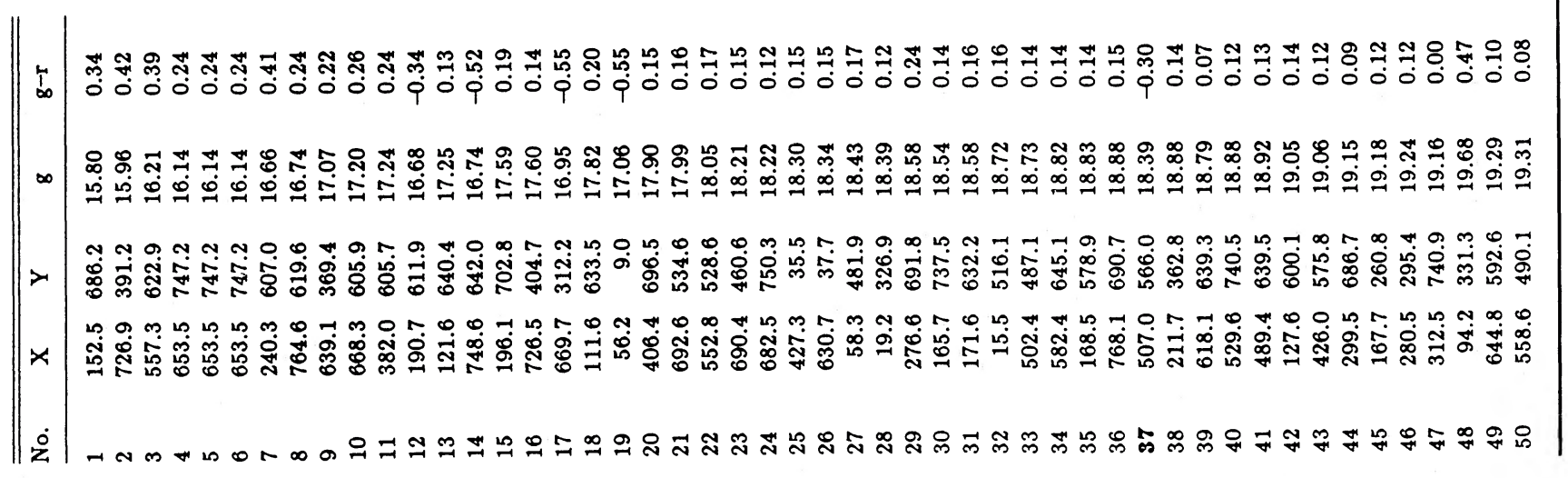




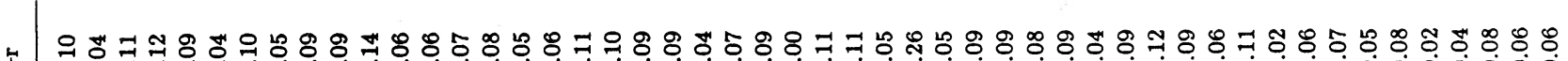
-

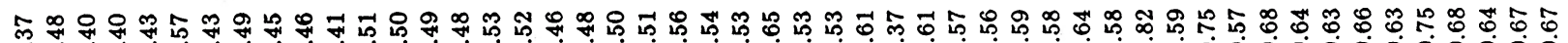

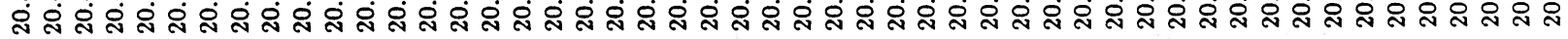

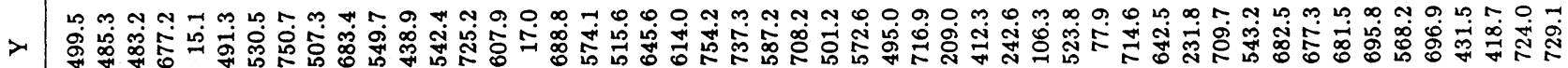
17.

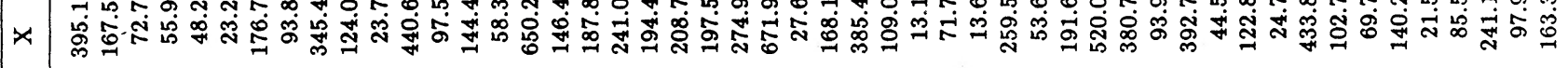

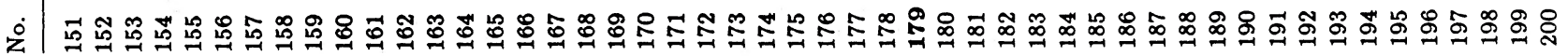

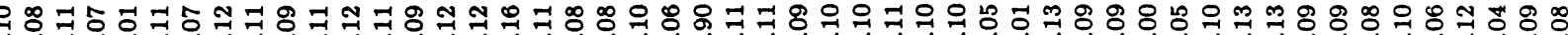

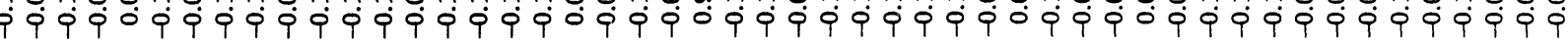

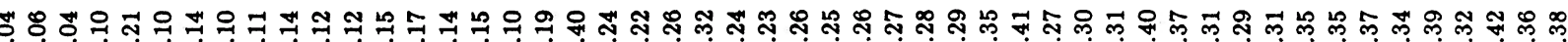

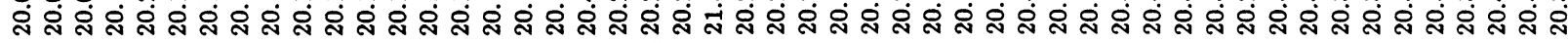
Or.

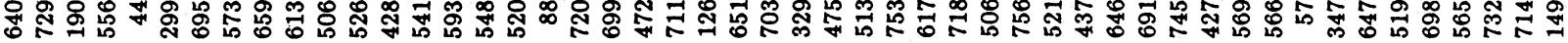
꾸 숭

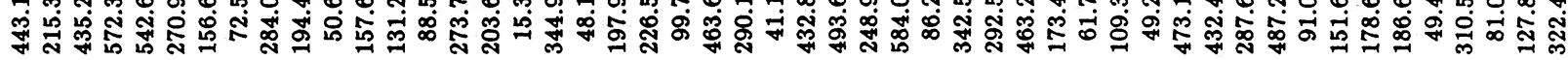

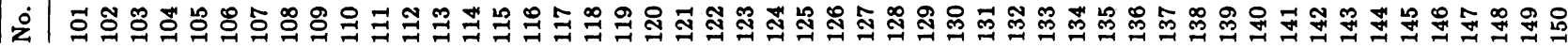

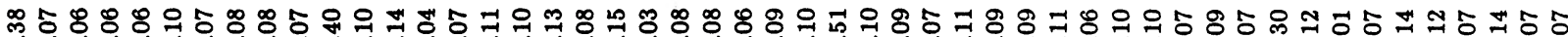

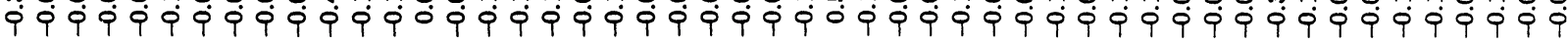

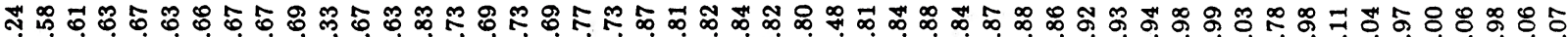

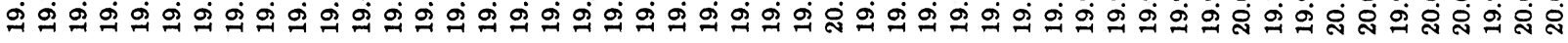

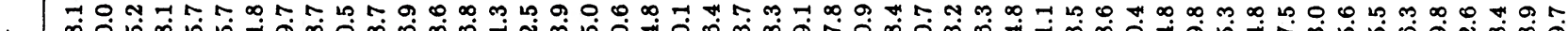

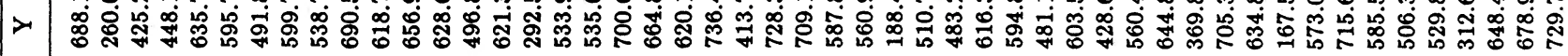

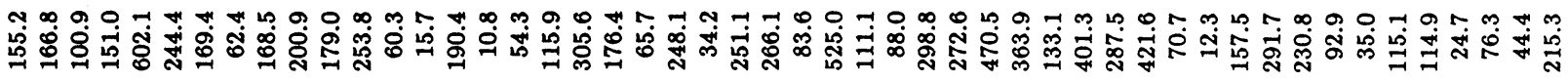

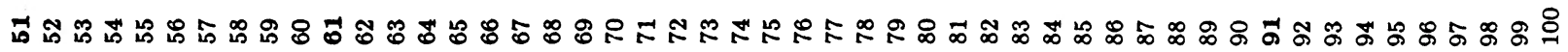

ז

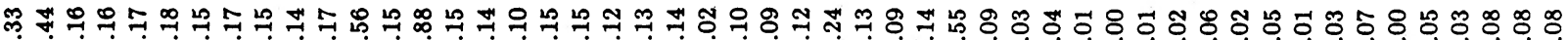
үi

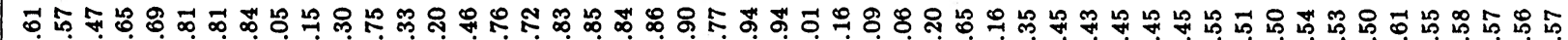

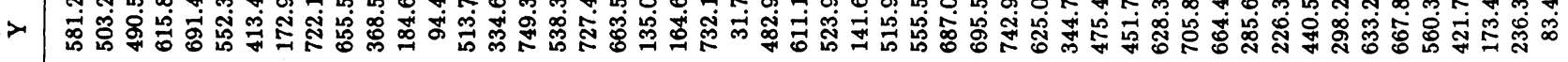
$\times$

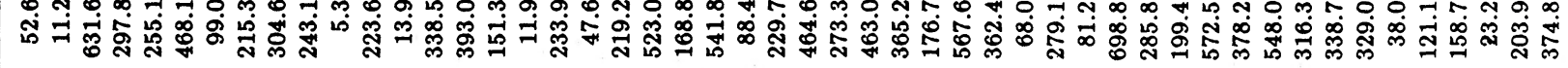
$\| \dot{z}$

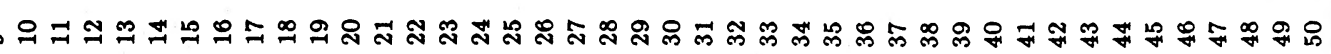




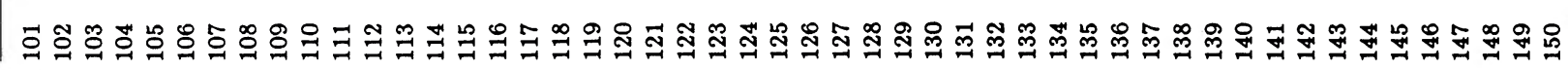

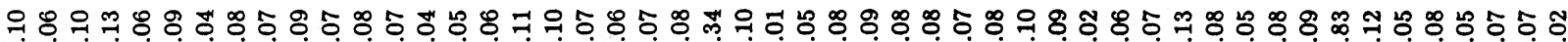
і́

웅우유.

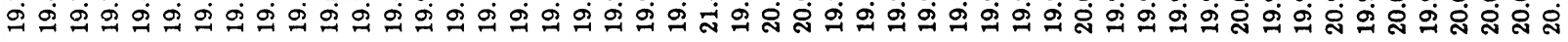
น

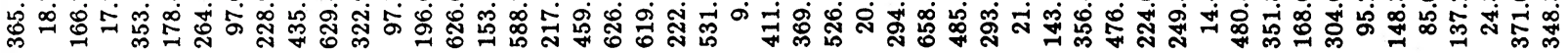

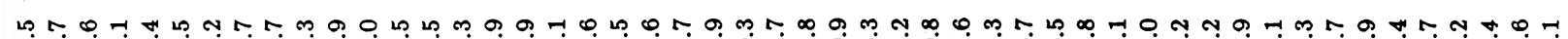

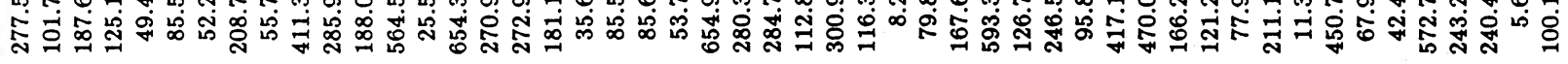

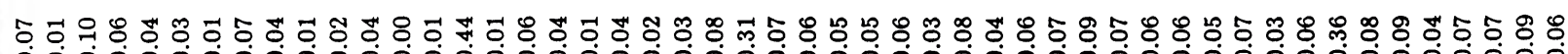

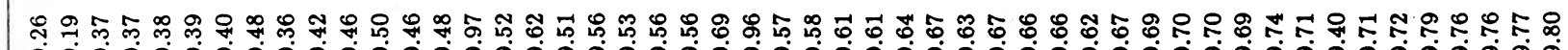

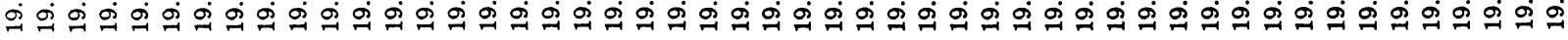

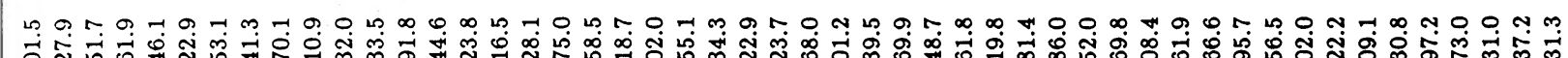

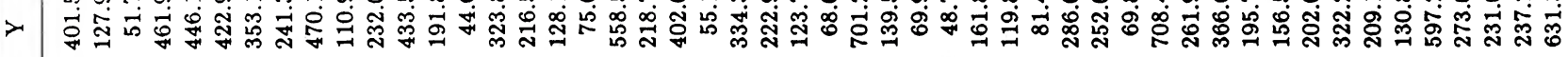

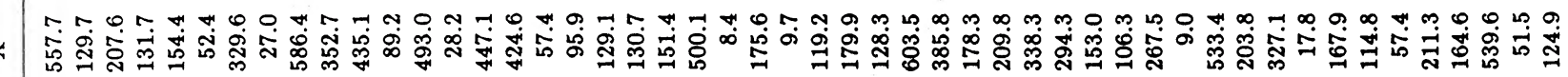

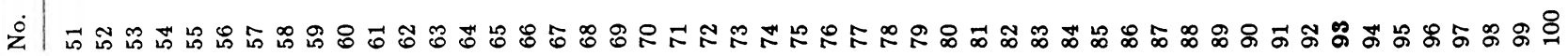

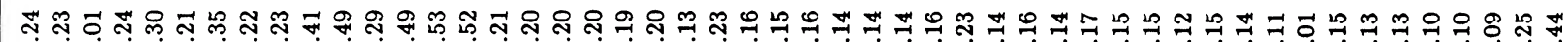

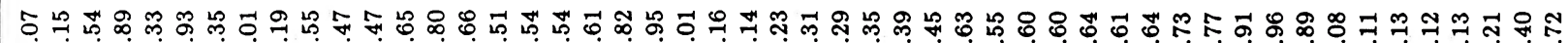

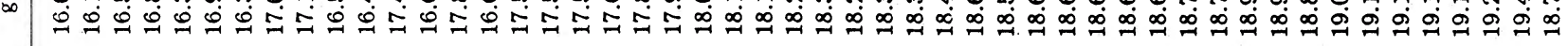

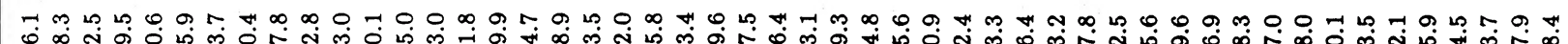
入 מ

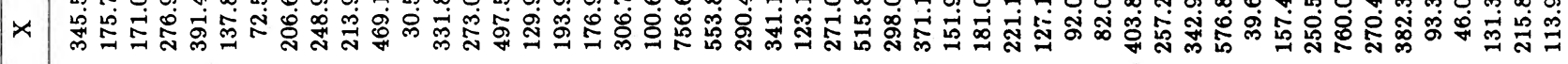
$\| \dot{0}$ 


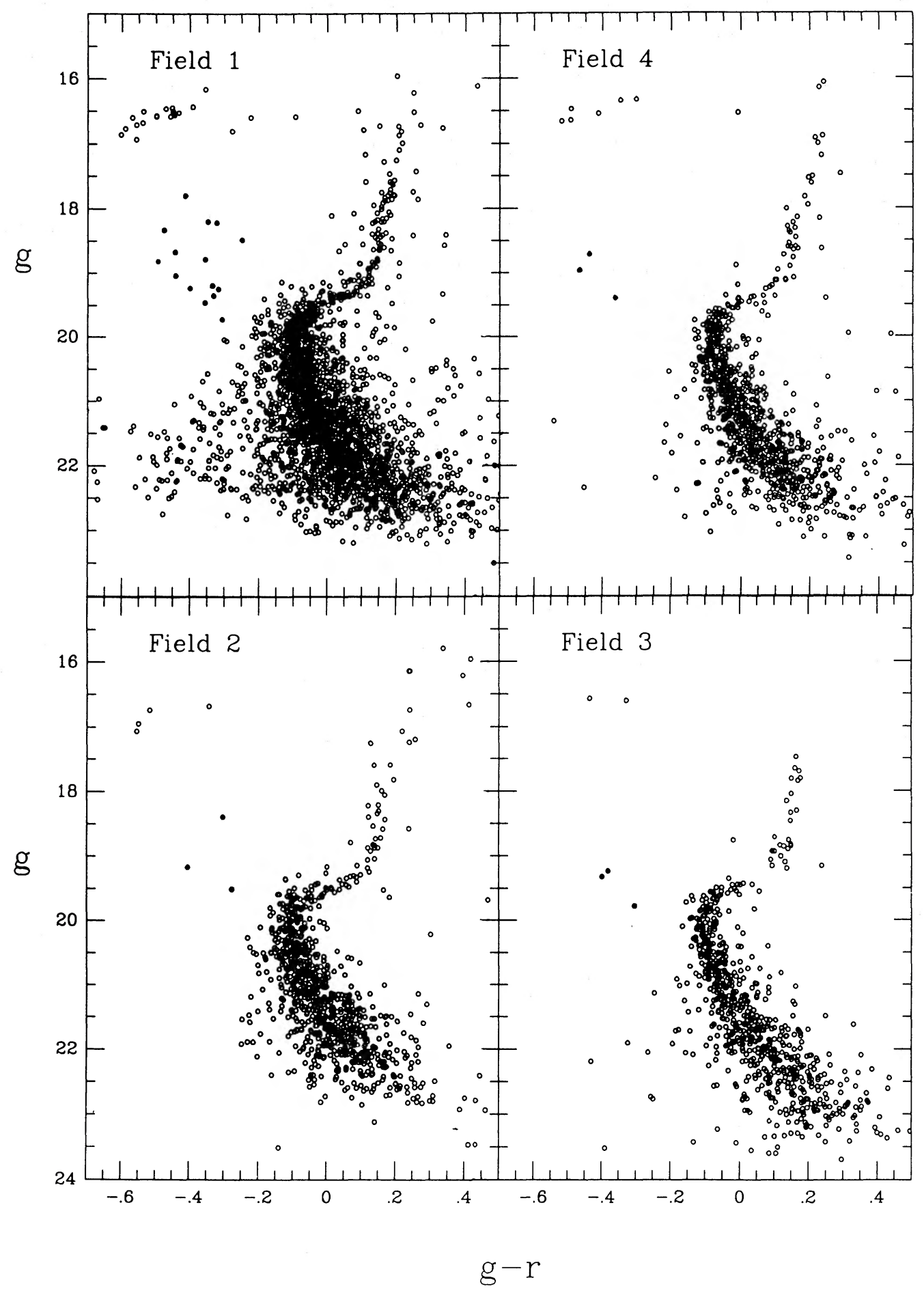

Fig. 2. $-C-M$ diagrams for the four NGC 5053 fields. The blue stragglers are plotted as solid points, and number 20 has been included in the field 4 diagram.

along the subgiant and main sequences were measured using these diagrams, and are listed in Table 4. The uncertainties in the colors are \pm 0.01 for stars brighter than $18 \mathrm{mag}, \pm 0.012$ for stars between 18 and $19 \mathrm{mag}, \pm 0.015$ for stars between 19 and $20 \mathrm{mag}, \pm 0.025$ for stars between 20 and $21 \mathrm{mag}, \pm 0.05$ for stars between 21 and $22 \mathrm{mag}$, and \pm 0.08 for stars between 22 and $23 \mathrm{mag}$. $A$ major feature seen in these $C-M$ diagrams, and the focus of the present paper, is the sequence of blue straggler stars (i.e., those stars along the luminous extension of the upper main sequence). No attempt has been made to "clean" the $C-M$ diagrams of possibly spurious points, except in the blue straggler region where great care was taken to eliminate points corresponding to stars with erroneous photometry.

Figure 3 shows the $C-M$ diagrams plotted as a function of projected angular distance from the cluster center, $R$ (arcsec). In computing $R$, the $\sim 15$ pixel interchip spacing between each 
TABLE 4

\begin{tabular}{|c|c|c|c|}
\hline \multicolumn{4}{|c|}{ Mean Ridge Line } \\
\hline $\begin{array}{c}g \\
(1)\end{array}$ & $\begin{array}{c}g-r \\
(2)\end{array}$ & $\begin{array}{c}g \\
(1)\end{array}$ & $\begin{array}{c}g-r \\
\text { (2) }\end{array}$ \\
\hline 17.0 . & 0.218 & $19.7 \ldots \ldots$ & -0.075 \\
\hline $17.2 \ldots$ & 0.212 & $19.8 \ldots$ & -0.086 \\
\hline $17.4 \ldots \ldots$ & 0.194 & $19.9 \ldots \ldots$ & -0.093 \\
\hline $17.6 \ldots$ & 0.181 & 20.0. & -0.093 \\
\hline $17.8 \ldots \ldots$ & 0.165 & $20.2 .$. & -0.091 \\
\hline $18.0 \ldots \ldots$ & 0.154 & $20.4 \ldots \ldots$ & -0.085 \\
\hline $18.2 \ldots \ldots$ & 0.152 & $20.6 \ldots \ldots$ & -0.077 \\
\hline $18.4 \ldots \ldots$ & 0.151 & $20.8 \ldots \ldots$ & -0.063 \\
\hline $18.6 \ldots \ldots$ & 0.149 & $21.0 \ldots \ldots$ & -0.049 \\
\hline $18.8 \ldots \ldots$ & 0.138 & $21.2 \ldots \ldots$ & -0.030 \\
\hline $19.0 \ldots \ldots$ & 0.118 & $21.4 \ldots \ldots$ & -0.007 \\
\hline $19.1 \ldots \ldots$ & 0.106 & $21.6 \ldots \ldots$ & +0.02 \\
\hline $19.2 \ldots$ & 0.094 & $21.8 \ldots \ldots$ & +0.05 \\
\hline $19.3 \ldots$ & 0.074 & $22.0 \ldots \ldots$ & +0.07 \\
\hline $19.4 \ldots \ldots$ & 0.035 & $22.2 \ldots \ldots$ & +0.11 \\
\hline $19.5 \ldots \ldots$ & -0.006 & $22.4 \ldots \ldots$ & +0.14 \\
\hline $19.6 \ldots \ldots$ & -0.046 & & \\
\hline
\end{tabular}

of the four chips was taken into account, and the distances were measured assuming that the cluster center is located in field 1 at the position of the isolated star east-north-east of blue straggler 2 (at $[X, Y]=[574,174])$. The radial bins $R \leq 105^{\prime \prime}$, $105^{\prime \prime} \leq R \leq 180^{\prime \prime}$, and $R \geq 180^{\prime \prime}$ were chosen to give approximately equal numbers of stars in each $C$ - $M$ diagram. In the diagrams, the 24 blue stragglers are shown as solid dots. Note that the most luminous blue stragglers tend to be located in the innermost field.

\section{b) Metal Abundance of NGC 5053}

The giant stars in NGC 5053 have metal abundances that are among the lowest of any stars known in the Galaxy. SKJ summarized the pre-1977 determinations of $[\mathrm{Fe} / \mathrm{H}]$ for stars in NGC 5053, and concluded that $[\mathrm{Fe} / \mathrm{H}]=-2.2$ (based on the mean period of the RR Lyrae stars, UV-excess measurements, and $[B-V]_{0, g}$, the well-known metallicity index equal to the dereddened color of the RGB at the level of the horizontal branch [Sandage and Smith 1966]). More recently, Bell and Gustafsson (1983) derived $[\mathrm{Fe} / \mathrm{H}]=-2.58$ from a comparison of synthetic spectra with Searle and Zinn's (1978) spectra of red giants, and Suntzeff, Kraft, and Kinman (1988) derived $[\mathrm{Fe} / \mathrm{H}]=-2.2$ from the spectroscopy of six giant stars. With our new photometry, it is possible to independently recompute $(B-V)_{0, g}$ (through a transformation equation relating $g-r$ to $B-V)$ and derive a new estimate of the mean metal abundance of the giant stars.

The observed color of the RGB at the level of the horizontal branch is $(g-r)=0.23 \pm 0.02 \mathrm{mag}$. The transformation equation $g-r=-0.351+0.863(B-V)$, derived using Kent's (1985) photometry of giant stars in M3 and M92, then gives $(B-V)_{g}=0.68 \pm 0.03$. Correcting for the interstellar reddening in the direction of NGC $5053\left(l=+336^{\circ}\right.$, $\left.b=+79^{\circ}\right)$ using the SKJ reddening value of $E(B-V)=0.01 \pm 0.02$ mag (which agrees with the Burstein and Heiles [1982] value for the extinction in the direction of the north Galactic pole), we calculate that $(B-V)_{0, g}=0.67$ $\pm 0.03 \mathrm{mag}$, which agrees precisely with the SKJ estimate for $(B-V)_{0, g}$. Thus the SKJ conclusion that NGC 5053 has one of the bluest $(B-V)_{0, g}$ values, and correspondingly, one of the lowest metal abundances of any known globular cluster, is recovered.

In the discussions that follow, we shall assume that $[\mathrm{Fe} / \mathrm{H}]=-2.3$, which corresponds to $Z=0.0001$ (assuming solar proportions of the elements). We will also assume that the mean helium abundance is $Y=0.25 \pm 0.04$ (Iben and Rood 1970; Kunth and Sargent 1983; Boesgaard and Steigman 1985), that $E(g-r) \sim E(B-V)$, and that the total extinction in the $g$-passband is $A(g)=3.29 E(B-V)($ Kent 1985).

\section{c) Distance Modulus and Age of NGC 5053}

SKJ derived a distance modulus of $(m-M)_{0}=16.03 \mathrm{mag}$ for NGC 5053. They observed that the magnitude level of the horizontal branch is at $V=16.63 \mathrm{mag}$, adopted a reddening $E(B-V)=0.01 \pm 0.02 \mathrm{mag}$, and assumed that the absolute visual magnitude for the RR Lyrae stars in the cluster is $\left\langle M_{V}(\mathrm{RR})\right\rangle=+0.60 \mathrm{mag}$. Because their photometry reached only to $\sim 17.3 \mathrm{mag}$, no attempt was made to derive the age of the cluster. With the photometry presented above, and the above estimates of the mean metal and helium abundance, a distance modulus and the age of NGC 5053 can be derived directly by fitting theoretical isochrones to the new observations.

Figure 4 compares a composite of the four $C-M$ diagrams shown in Figure 2, with theoretical isochrones computed by Bell and VandenBerg (1987). These isochrones were computed specifically for the Thuan-Gunn system, and assume a metal abundance $Z=0.0001$, and a ratio of mixing length to pressure scale height of $\alpha=1.60$. The isochrones were shifted vertically by $\Delta g=16.13 \mathrm{mag}$ to achieve the best alignment with the upper main sequence and subgiant branch for NGC 5053. On the left, $Y=0.20$ isochrones are plotted for ages of $8,10,12,14$, 16 , and $18 \mathrm{Gyr}$; and, on the right, $Y=0.30$ isochrones for ages $6,8,10,12,14$, and 16 Gyr are plotted. [The present study is one of the first to use these recently computed Thuan-Gunn isochrones. Previous studies, such as Cohen (1985) and Nemec and Harris (1987), used isochrones computed for the $U B V$ system that were transformed to the $(g, g-r)$-plane].

In Figure 4, the isochrones that provide the best vertical fit to the observed main-sequence turnoff region suggest that the distance modulus is $(m-M)_{0}=16.15 \pm 0.05$ mag. Although small redward color shifts of the $Y=0.20$ and 0.30 isochrones would improve the fits to the lower main-sequence (the required shift is larger for the $Y=0.30$ isochrones), no ad hoc color shifts have been made. ${ }^{1}$ In both panels of Figure 4 the isochrone corresponding to an age of $\sim 18$ Gyr fits the observations best. (Note that the oldest isochrone in the $Y=0.30$ panel corresponds to an age of $16 \mathrm{Gyr}$, and an extrapolation to 18 Gyr was made). Since the small color shifts mentioned above have little effect on these age estimates, it follows that for the assumed mean helium abundance $(Y=0.25)$ the best estimate of the cluster age is $18 \mathrm{Gyr}$, with an estimated uncertainty of \pm 3 Gyr. For the cluster to be younger than this, the distance modulus would have to be increased. However, for younger ages the goodness of the fit of the isochrones becomes progressively worse in the main-sequence turnoff region. The mainsequence turnoff mass for NGC 5053, $M_{\text {turnoff }}$, is

\footnotetext{
${ }^{1}$ Historically, redward shifts of theoretical isochrones have been needed to match the observed $B V$ main-sequence turnoffs. The shifts have sometimes been as much as $\Delta(B-V)=0.10$ mag (e.g., Sandage 1983; Heasley and Christian 1986; Smith et al. 1986).
} 


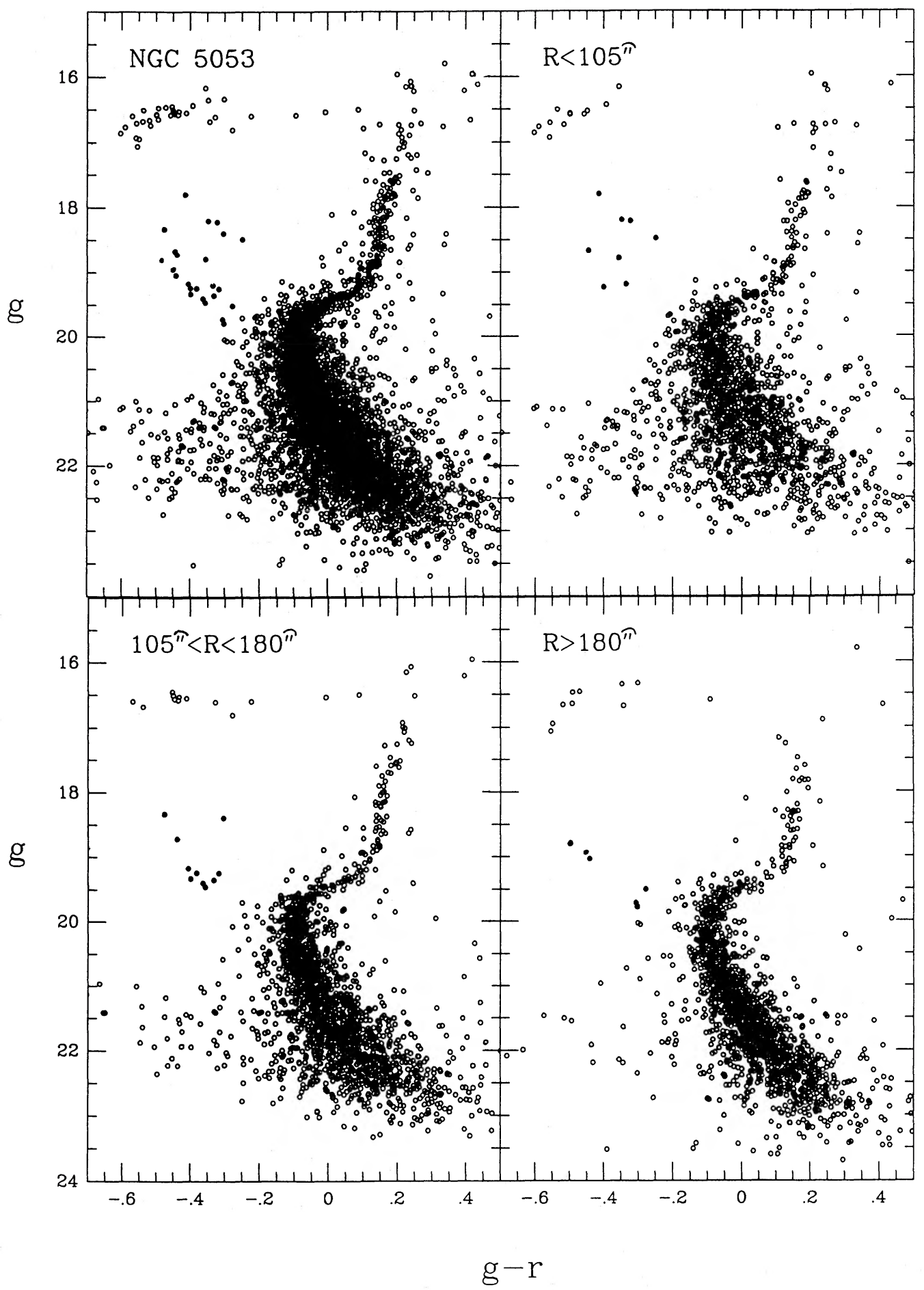

FIG. 3.-Color-magnitude diagrams for NGC 5053, plotted for three different radial groupings of the stars: (upper left) Composite diagram with 5919 stars; (upper right) 1888 stars within $105^{\prime \prime}$ of the cluster center; (lower left) 2169 stars in the annulus $105^{\prime \prime} \leq R \leq 180^{\prime \prime}$; (lower right) 1862 stars more distant than $180^{\prime \prime}$ from the cluster center. Blue straggler 20, which was not measured on the 4-shooter frames (because of its great distance from the cluster center), has been included in this panel. 

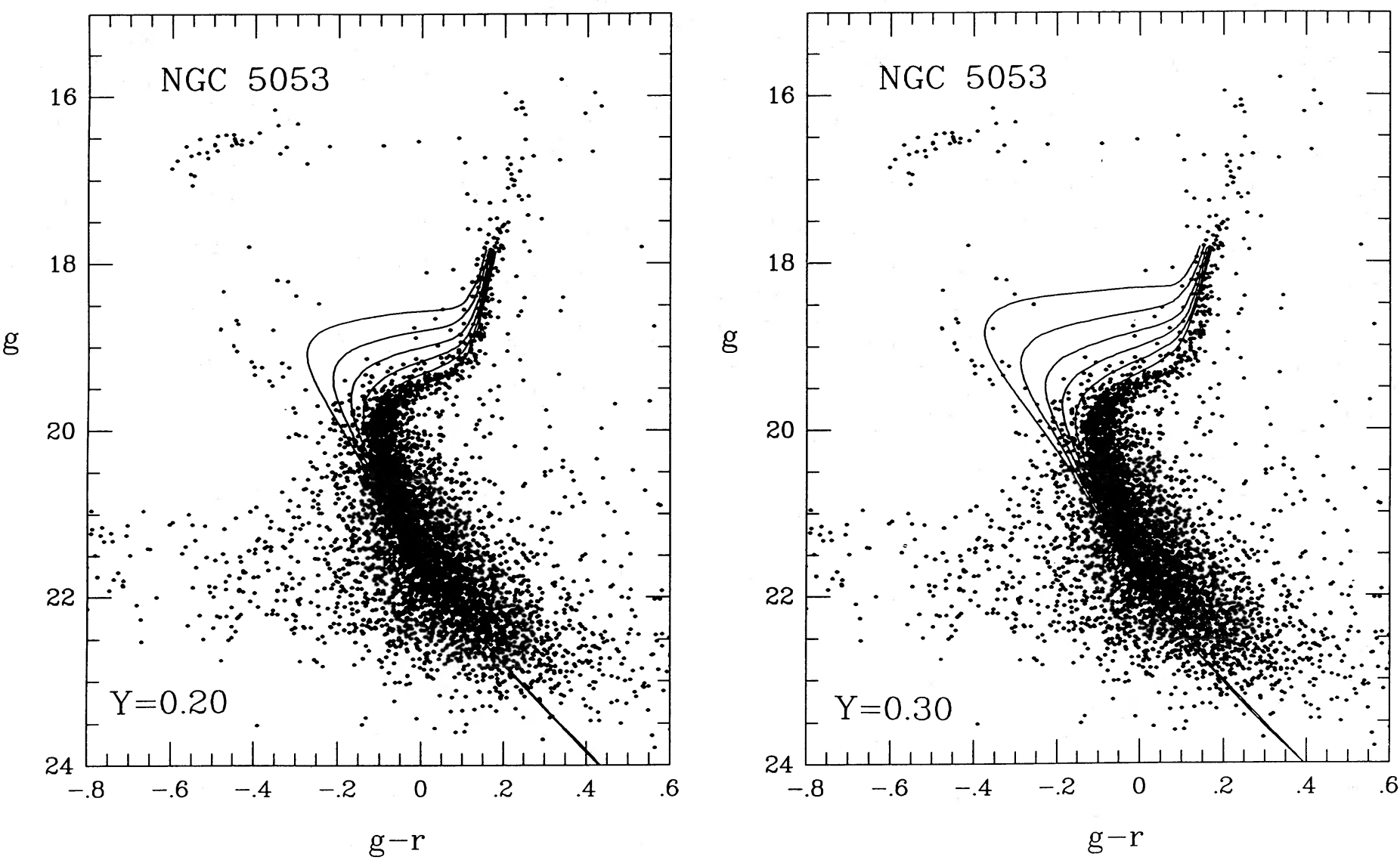

FIG. 4-Composite color-magnitude diagram for NGC 5053, compared with theoretical isochrones computed by Bell and VandenBerg (1987). (left panel) The isochrones for $Y=0.20,[\mathrm{Fe} / \mathrm{H}]=-2.2$, and for the ages $8,10,12,14,16$, and $18 \mathrm{Gyr}$. (right panel) The isochrones for $Y=0.30$, $[\mathrm{Fe} / \mathrm{H}]=-2.2$, and for the ages 6 , $8,10,12,14$, and $16 \mathrm{Gyr}$.

approximately $0.80 M_{\odot}$ [the exact value depends on the assumed stellar evolution model. If $Y=0.20$ the Bell and VandenBerg model predicts $M_{\text {turnoff }}=0.786 M_{\odot}(18 \mathrm{Gyr}), 0.812$ $M_{\odot}(16 \mathrm{Gyr})$ and $0.842 M_{\odot}(14 \mathrm{Gyr})$; and, if $Y=0.30$ the model predicts $0.689 M_{\odot}(16 \mathrm{Gyr})$ and $\left.0.713 M_{\odot}(14 \mathrm{Gyr})\right]$.

Our conclusion that NGC 5053 is very old is supported by the observed magnitude difference between the horizontal branch and the main-sequence turnoff point (defined as the bluest point on the main sequence). The mean level of the NGC 5053 horizontal branch (HB) is at $g=16.45 \pm 0.10 \mathrm{mag}$, and the bluest point of the main sequence (TO) is at $(g, g-r)$ $=19.95 \pm 0.10 \mathrm{mag},-0.095 \pm 0.01 \mathrm{mag}$ ), giving a difference $\Delta g(\mathrm{TO}-\mathrm{HB})=3.50 \pm 0.07 \mathrm{mag}$. If this difference can be directly compared with $V$ magnitude differences for nearby globular clusters (and the transformation between the two photometric systems suggests that it can), then the similarity to the fiducial value of $\Delta V=3.50 \mathrm{mag}$ for old globular clusters (Sandage 1970; see also Renzini and Fusi Pecci 1988) supports the conclusion above that NGC 5053 has a great age.

With the above value for the mean level of the NGC 5053 horizontal branch, and assuming $\left\langle M_{g}(\mathrm{RR})\right\rangle=0.50 \pm 0.23$ mag for the absolute magnitude of the RR Lyrae stars [corresponding to $\left\langle M_{V}(\mathrm{RR})\right\rangle=0.60 \pm 0.20 \mathrm{mag}$ ], we derive, as a second estimate for the NGC 5053 distance modulus, the value $(m-M)_{0}=15.95 \pm 0.18 \mathrm{mag}$. Combining this distance determination with that derived above from the vertical shift of the theoretical isochrones to the main-sequence turnoff, we conclude that, according to our data, the best estimate of the distance modulus is $(m-M)_{0}=16.05 \pm 0.14 \mathrm{mag}$, in agreement with the SKJ value.

\section{d) Subgiant and Upper-Main-Sequence Luminosity Function}

Star counts based on the stars that were used to construct the composite $C-M$ diagram in Figure 3 are given in Table 5, for magnitude intervals of $\Delta g=0.50 \mathrm{mag}$. Although these counts include all the stars in Figure 3, the results would be approximately the same if we had restricted attention to only the subgiants and main-sequence stars. Because of the extreme openness of NGC 5053, and its high Galactic latitude, no corrections for spurious points resulting from image crowding, or for background galaxies, were made. The luminosity function

TABLE 5

LUMINOSITY FUNCTION

\begin{tabular}{cr|cr}
\hline \hline $\begin{array}{c}g \text { Range } \\
(1)\end{array}$ & $\begin{array}{c}N(g) \\
(2)\end{array}$ & $\begin{array}{c}g \text { Range } \\
(1)\end{array}$ & $\begin{array}{c}N(g) \\
(2)\end{array}$ \\
\hline $16.0-16.5 \ldots \ldots$ & 7 & $20.0-20.5 \ldots \ldots$ & 557 \\
$16.5-17.0 \ldots \ldots$ & 13 & $20.5-21.0 \ldots \ldots$ & 756 \\
$17.0-17.5 \ldots \ldots$ & 14 & $21.0-21.5 \ldots \ldots$ & 853 \\
$17.5-18.0 \ldots \ldots$ & 36 & $21.5-22.0 \ldots \ldots$. & 928 \\
$18.0-18.5 \ldots \ldots$ & 46 & $22.0-22.5 \ldots \ldots$. & 786 \\
$18.5-19.0 \ldots \ldots$ & 60 & $22.5-23.0 \ldots \ldots$ & 321 \\
$19.0-19.5 \ldots \ldots$. & 150 & $23.0-23.5 \ldots \ldots$ & 51 \\
$19.5-20.0 \ldots \ldots$ & 396 & & \\
\hline
\end{tabular}


$\Phi(g)=$ the number of stars in a magnitude interval $g-0.25$ to $g+0.25$, is plotted against $g$ in Figure 5. Clearly, for magnitudes brighter than $g=21$ and fainter than $g=19$, the luminosity function is steep, with slope $\Delta \Phi(g) / \Delta g=388$, and shows no sign of turning over (the slope of the luminosity function would be even larger if a small correction for crowding had been made; between $g=21$ and 22 the slope becomes shallower, but the counts continue to rise; and fainter than $g=22$ the luminosity function turns over sharply. The turnover at $g \sim 22$ is attributed to the fact that the limiting magnitudes of the short 4-Shooter exposures are being reached. The steep slope seen for $19 \leq g \leq 21 \mathrm{mag}$ is similar to the steep slopes seen in the $\Phi\left(M_{V}\right)$ plot for the low metal abundance globular clusters M15 and M68 (McClure et al. 1986), which have been taken to be indicative of a steep mass function. Obviously, our luminosity function represents a preliminary result since it is based entirely on short exposures and therefore is estimated to be complete to only $g=21$ (which corresponds to $M_{g} \sim 5$ mag). A more accurate luminosity function, based on PSF photometry to 26 mag obtained using long-exposure CCD frames taken with the CFH $3.6 \mathrm{~m}$ telescope, is in preparation (Fahlman, Richer, and Nemec 1989).

\section{BLUE STRAGGLERS IN NGC 5053}

Renzini, Mengel and Sweigart (1977, hereafter RMS) predicted that if blue straggler stars are mass transfer binary systems, then a system as diffuse as NGC 5053 provides an ideal environment for harboring binary blue stragglers, and their suspected progeny, the anomalous Cepheids. This prediction was based, in part, on the calculations by Hills and Day
(1976), which showed that the probability is very low that even one stellar encounter would occur in a cluster as open NGC $5053 .{ }^{2}$ In this section we identify the population of blue stragglers in NGC 5053 and study some of their properties.

\section{a) Identification and Completeness}

A total of 35 candidate blue stragglers, with $17.4 \leq g \leq 20.0$ mag, and $-0.6 \leq g-r \leq-0.2 \mathrm{mag}$, was identified from the 4-Shooter $C$ - $M$ diagrams. Subsequently, 11 of these were found to be stars other than blue stragglers and only appeared to lie in the blue straggler region of the $C-M$ diagram because of errors in their photometric colors (caused mainly by image crowding). The challenge of assembling a complete list of bona fide blue straggler stars in NGC 5053 was to eliminate such spurious candidates. In general, the same methods that were used in Paper I to identify blue stragglers in NGC 5466 were employed here. The identifications relied heavily on the visual inspection of the stellar images and their environs, both before and after subtraction of the PSF. A less subjective evaluation of the photometry, using the DAOPHOT statistics CHI and SHARP (to judge the "goodness of the fit" of the PSFs to the stellar images), was also made. In addition, four independent $B V C$ - $M$ diagrams were constructed from CFH CCD frames

\footnotetext{
${ }^{2}$ The globular cluster evolution model of Chernoff and Shapiro (1987), which evaluates the relative importance of relaxation, stellar mass loss, heating, and mass loss following passage through the Galactic disk, and mass loss across a tidal boundary, shows that globular clusters which were originally very open are now even less centrally concentrated than in the past. Consequently, the present-day frequency of stellar encounters is lower than in the past. In very open systems like NGC 5053 these effects probably are not large enough to change significantly the main conclusions of RMS.
}

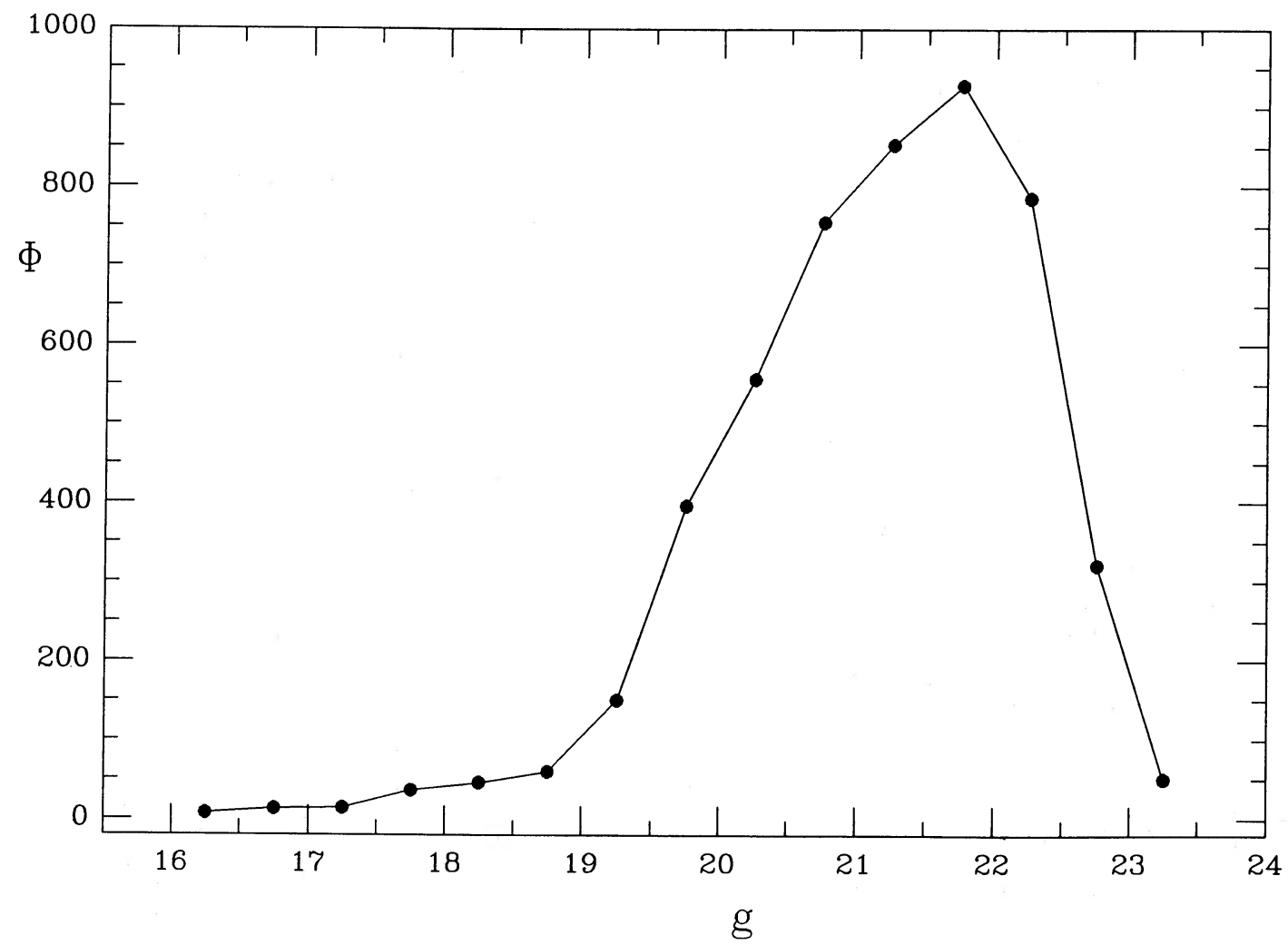

Fig. 5.-The observed luminosity function for NGC 5053. $\Phi(g)$ is the number of stars in $\Delta g=0.5$ mag intervals between $g=16.0$ and $23.5 \mathrm{mag}$. 
(Fahlman, Richer, and Nemec 1989) taken under excellent seeing conditions (FWHM $\sim 0$ ".80), and three pairs of CCD frames taken in 1986 with the Palomar 60 inch $(1.5 \mathrm{~m})$ telescope (hereafter P60). Figure 6 (Plate 23) is a photograph of the central region of NGC 5053 made from one of these frames, and Figure 7 (Plate 24) is a reproduction of one of the P60 frames.

The final list of 24 probable blue straggler stars is given in Table 6, and the stars are identified in Figures 1, 6, and 7. For each star Table 6 contains: the $X$ - and $Y$-coordinates; the number of the 4-Shooter field in which it was found; the $g$ and $g-r$ photometry, and the associated uncertainties in the photometry; the image shape parameters, CHI and SHARP (see below); the projected distance from the cluster center, $R$ ( $\operatorname{arcsec}$ ); and a weight, equal to the number of times the star was independently identified as a blue straggler in the various $C-M$ diagrams. Measurement of the pair of CFH frames recovered the blue stragglers $1,2,3,4,7$, and 13, all of which previously had been discovered on the 4-Shooter frames. Blue straggler 20 , because of its great distance from the cluster center, was measured only on the P60 frames. The three $C-M$ diagrams constructed from the P60 frames recovered blue straggler 9 (one time), 10 (one time), 14 (two times), 15 (two times), 19 (one time), 20 (three times), and 21 (three times).

For each measured star, DAOPHOT computes the image shape parameters $\mathrm{CHI}$ and SHARP. The CHI values correspond to "the ratio of the observed pixel-to-pixel scatter from the model image profile, divided by the expected pixel-to-pixel scatter from the image profile," and the SHARP value is an image statistic "vaguely related to the intrinsic (i.e., outside the atmosphere) angular size of the astronomical object" (Stetson 1986). If the images of the stars being measured are single, and the photometric parameters are set correctly (i.e., if the readout noise and the photons $\mathrm{ADU}^{-1}$ are given correctly when DAOPHOT is run), the CHI values should be distributed about 1.0, and the SHARP values should be distributed about 0.0 .

Figures 8 and 9 show the distributions of the $\mathrm{CHI}$ and SHARP values, respectively, plotted as a function of $g$ magnitude, for all the stars measured on the 4-Shooter frames. The CHI values are distributed about 1.0 for stars fainter than $g \sim 20$ mag, but deviate systematically, in the mean, for brighter stars. Brighter than 20 mag the $\mathrm{CHI}$ values tend to increase approximately linearly with magnitude. Since all the measured stars that are bright have relatively large CHI values and most of these are clearly cluster subgiants and horizontal branch stars, the large CHI values determined for some of the blue stragglers (Table 6) do not necessarily mean that their images are nonstellar or that their photometry is poor. In fact, the narrowness of the observed giant branch and the upper main-sequence indicates that the photometry at the magnitude level of the blue stragglers must be fairly precise. ${ }^{3}$ The SHARP values show no trend with magnitude and appear to be distributed about the expected value of 0.0 .

The completeness of the list of blue stragglers is estimated to be $\gtrsim 90 \%$ in the area surveyed, and falls off near the center where the crowding is greatest, and at low luminosities where the blue straggler sequence asymptotically approaches the main sequence. This estimate of the completeness is based on the fact that the center of NGC 5053 is considerably less

\footnotetext{
${ }^{3}$ The most likely cause of the linear trend in the $\mathrm{CHI}$ values is that it is related to the PSF variations on the 4-Shooter frames. The same dependence on magnitude of CHI values is also present in unpublished photometry from other 4-Shooter frames (Nemec 1989). A less likely explanation is that there are small residual flat fielding problems with the 4-Shooter frames. Other much less likely explanations for the trend might be that the values for the photon $\mathrm{ADU}^{-1}$ ratio and readout noise (recorded in Table 2) used by DAOPHOT were incorrect, or that image saturation occurred at $g \sim 20$ mag (corresponding to maximum ADU values per star $\sim 4000$ ), and not $\sim 18 \mathrm{mag}$, as assumed.
}

TABLE 6

Blue StRAGglers IN NGC 5053

\begin{tabular}{|c|c|c|c|c|c|c|c|c|c|c|c|}
\hline $\begin{array}{c}\text { Number } \\
\text { (1) }\end{array}$ & $\begin{array}{l}X \\
(2)\end{array}$ & $\begin{array}{c}Y \\
(3)\end{array}$ & $\begin{array}{l}\text { Field } \\
(4)\end{array}$ & $\begin{array}{c}g \\
(5)\end{array}$ & $\begin{array}{c}g-r \\
(6)\end{array}$ & $\begin{array}{l}\epsilon(r) \\
(7)\end{array}$ & $\begin{array}{c}\epsilon(g) \\
(8)\end{array}$ & $\begin{array}{c}\text { CHI } \\
(9)\end{array}$ & $\begin{array}{l}\text { SHARP } \\
(10)\end{array}$ & $\begin{array}{c}R \\
(11)\end{array}$ & $\begin{array}{c}\text { Weight } \\
\text { (12) }\end{array}$ \\
\hline $\begin{array}{l}1 \ldots \ldots \ldots \\
2 \ldots \ldots \ldots \\
3 \ldots \ldots \ldots \\
4 \ldots \ldots \ldots \\
5 \ldots \ldots \ldots\end{array}$ & $\begin{array}{l}450.5 \\
590.7 \\
645.0 \\
364.1 \\
465.9\end{array}$ & $\begin{array}{r}201.3 \\
87.6 \\
147.7 \\
239.2 \\
401.8\end{array}$ & $\begin{array}{l}1 \\
1 \\
1 \\
1 \\
1\end{array}$ & $\begin{array}{l}17.80 \\
18.19 \\
18.48 \\
18.22 \\
18.67\end{array}$ & $\begin{array}{l}-0.41 \\
-0.35 \\
-0.25 \\
-0.32 \\
-0.44\end{array}$ & $\begin{array}{l}0.01 \\
0.01 \\
0.01 \\
0.01 \\
0.01\end{array}$ & $\begin{array}{l}0.01 \\
0.01 \\
0.01 \\
0.01 \\
0.01\end{array}$ & $\begin{array}{l}3.34 \\
2.94 \\
2.58 \\
1.83 \\
1.33\end{array}$ & $\begin{array}{r}-0.44 \\
0.01 \\
0.18 \\
-0.07 \\
-0.19\end{array}$ & $\begin{array}{r}52^{\prime \prime} \\
8 \\
27 \\
82 \\
104\end{array}$ & $\begin{array}{l}2 \\
2 \\
2 \\
2 \\
1\end{array}$ \\
\hline $\begin{array}{c}6 \ldots \ldots \ldots \\
7 \ldots \ldots \ldots \\
8 \ldots \ldots \ldots \\
9 \ldots \ldots \ldots \\
10 \ldots \ldots \ldots\end{array}$ & $\begin{array}{l}573.4 \\
318.4 \\
676.4 \\
717.4 \\
738.5\end{array}$ & $\begin{array}{l}433.3 \\
145.6 \\
668.7 \\
665.3 \\
261.4\end{array}$ & $\begin{array}{l}1 \\
1 \\
1 \\
1 \\
1\end{array}$ & $\begin{array}{l}18.33 \\
19.19 \\
19.04 \\
18.76 \\
18.79\end{array}$ & $\begin{array}{l}-0.47 \\
-0.33 \\
-0.44 \\
-0.48 \\
-0.35\end{array}$ & $\begin{array}{l}0.01 \\
0.01 \\
0.01 \\
0.02 \\
0.01\end{array}$ & $\begin{array}{l}0.01 \\
0.01 \\
0.01 \\
0.02 \\
0.01\end{array}$ & $\begin{array}{l}1.71 \\
1.11 \\
2.10 \\
3.07 \\
2.43\end{array}$ & $\begin{array}{r}-0.21 \\
-0.02 \\
0.27 \\
0.52 \\
0.27\end{array}$ & $\begin{array}{r}109 \\
85 \\
189 \\
191 \\
75\end{array}$ & $\begin{array}{l}1 \\
2 \\
1 \\
2 \\
2\end{array}$ \\
\hline $\begin{array}{l}11 \ldots \ldots \ldots \\
12 \ldots \ldots \ldots \\
13 \ldots \ldots \ldots \\
14 \ldots \ldots \ldots \\
15 \ldots \ldots \ldots\end{array}$ & $\begin{array}{l}613.6 \\
167.8 \\
674.9 \\
155.2 \\
179.0\end{array}$ & $\begin{array}{l}442.5 \\
322.2 \\
121.9 \\
688.1 \\
618.7\end{array}$ & $\begin{array}{l}1 \\
4 \\
1 \\
3 \\
3\end{array}$ & $\begin{array}{l}19.35 \\
19.40 \\
19.23 \\
19.24 \\
19.33\end{array}$ & $\begin{array}{l}-0.33 \\
-0.36 \\
-0.40 \\
-0.38 \\
-0.40\end{array}$ & $\begin{array}{l}0.01 \\
0.05 \\
0.01 \\
0.01 \\
0.01\end{array}$ & $\begin{array}{l}0.01 \\
0.05 \\
0.02 \\
0.01 \\
0.01\end{array}$ & $\begin{array}{l}1.15 \\
2.03 \\
1.36 \\
1.83 \\
1.02\end{array}$ & $\begin{array}{r}-0.12 \\
0.05 \\
0.22 \\
-0.06 \\
0.01\end{array}$ & $\begin{array}{r}113 \\
140 \\
34 \\
134 \\
153\end{array}$ & $\begin{array}{l}1 \\
1 \\
2 \\
3 \\
3\end{array}$ \\
\hline $\begin{array}{l}16 \ldots \ldots \ldots \\
17 \ldots \ldots \ldots \\
18 \ldots \ldots \ldots \\
19 \ldots \ldots \ldots \\
20 \ldots \ldots \ldots\end{array}$ & $\begin{array}{c}291.7 \\
406.3 \\
605.5 \\
80.6 \\
\ldots\end{array}$ & $\begin{array}{c}167.5 \\
647.0 \\
292.7 \\
443.4 \\
\ldots\end{array}$ & $\begin{array}{c}3 \\
2 \\
2 \\
1 \\
\cdots\end{array}$ & $\begin{array}{l}19.78 \\
19.17 \\
19.51 \\
19.72 \\
18.97\end{array}$ & $\begin{array}{l}-0.30 \\
-0.41 \\
-0.28 \\
-0.31 \\
-0.46\end{array}$ & $\begin{array}{l}0.01 \\
0.01 \\
0.01 \\
0.02 \\
0.02\end{array}$ & $\begin{array}{l}0.01 \\
0.01 \\
0.01 \\
0.03 \\
0.03\end{array}$ & $\begin{array}{c}1.29 \\
1.38 \\
1.50 \\
1.29 \\
\ldots\end{array}$ & $\begin{array}{c}-0.04 \\
-0.11 \\
-0.26 \\
-0.23 \\
\ldots\end{array}$ & $\begin{array}{r}288 \\
97 \\
197 \\
198 \\
555\end{array}$ & $\begin{array}{l}1 \\
1 \\
1 \\
1 \\
3\end{array}$ \\
\hline $\begin{array}{l}21 \ldots \ldots \ldots \\
22 \ldots \ldots \ldots \\
23 \ldots \ldots \ldots \\
24 \ldots \ldots \ldots\end{array}$ & $\begin{array}{l}113.9 \\
251.5 \\
230.7 \\
506.9\end{array}$ & $\begin{array}{r}8.4 \\
204.7 \\
143.8 \\
566.0\end{array}$ & $\begin{array}{l}4 \\
1 \\
1 \\
2\end{array}$ & $\begin{array}{l}18.72 \\
19.24 \\
19.46 \\
18.39\end{array}$ & $\begin{array}{l}-0.44 \\
-0.32 \\
-0.36 \\
-0.30\end{array}$ & $\begin{array}{l}0.01 \\
0.07 \\
0.04 \\
0.05\end{array}$ & $\begin{array}{l}0.01 \\
0.07 \\
0.04 \\
0.05\end{array}$ & $\begin{array}{l}1.67 \\
2.62 \\
1.83 \\
3.37\end{array}$ & $\begin{array}{l}0.13 \\
0.47 \\
0.11 \\
0.42\end{array}$ & $\begin{array}{l}107 \\
111 \\
114 \\
109\end{array}$ & $\begin{array}{l}4 \\
1 \\
1 \\
1\end{array}$ \\
\hline
\end{tabular}




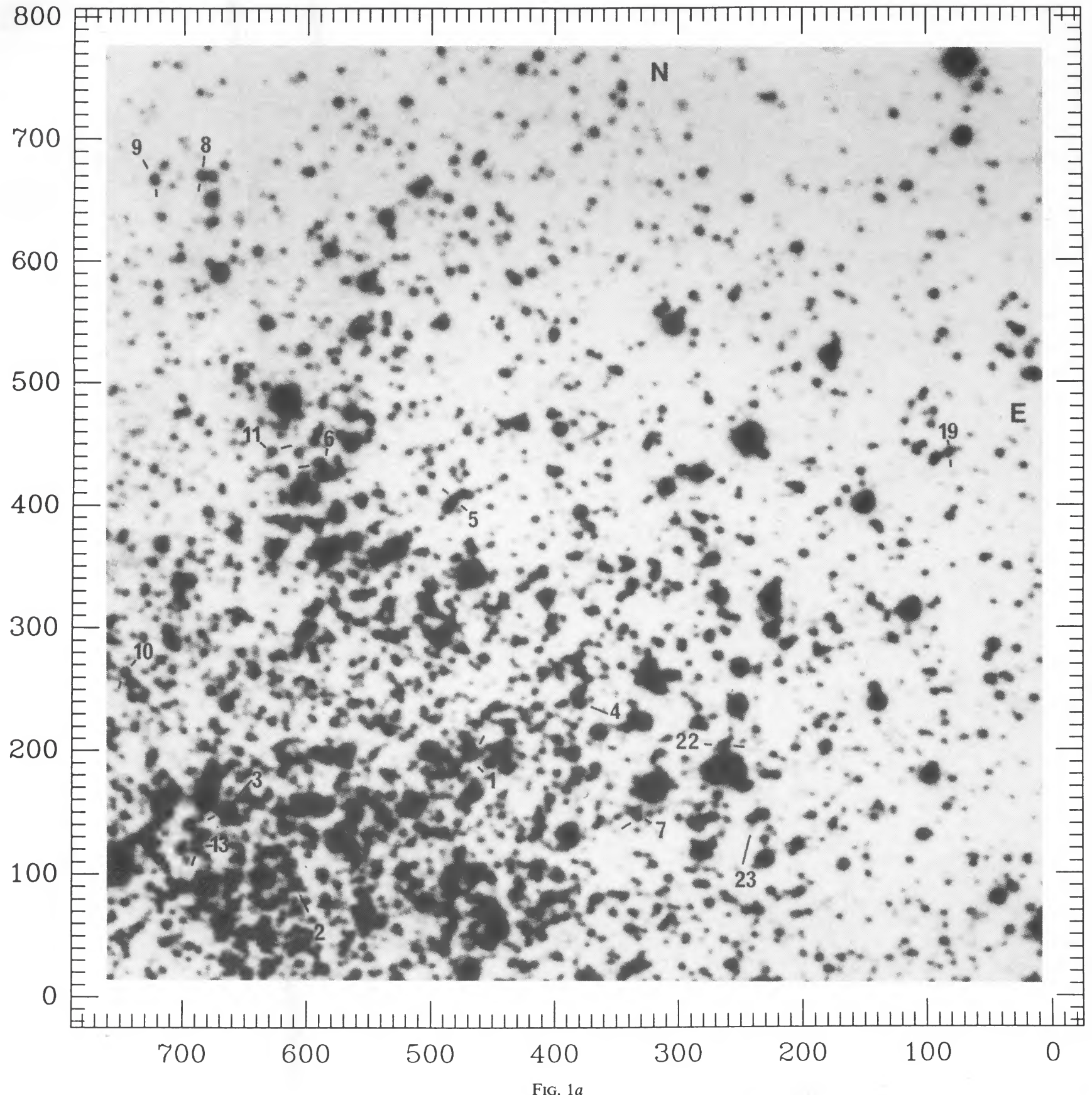

Fig. 1. (a) Photograph of the northeast quadrant (field 1) in NGC 5053. The reproductions for this field, and for the other three quadrants, are from the 4-Shooter CCD frames taken through the $r$-filter. The axes correspond to the $X$ - and $Y$-coordinates on the final trimmed frames, and the scale is $0^{\prime \prime} 33$ pixel ${ }^{-1}$. Blue stragglers $1,2,3,4,5,6,7,8,9,10,11,13,19,22$, and 23 are identified. (b) Photograph of the southeast quadrant (field 2) in NGC 5053. Blue stragglers 17, 18, and 24 are identified. (c) Photograph of the southwest quadrant (field 3) in NGC 5053. Blue stragglers 14, 15, and 16 are identified. (d) Photograph of the northeast quadrant (field 4) in NGC 5053. Blue stragglers 12 and 21 are identified.

Nemic AND CoHEN (see 336, 791) 


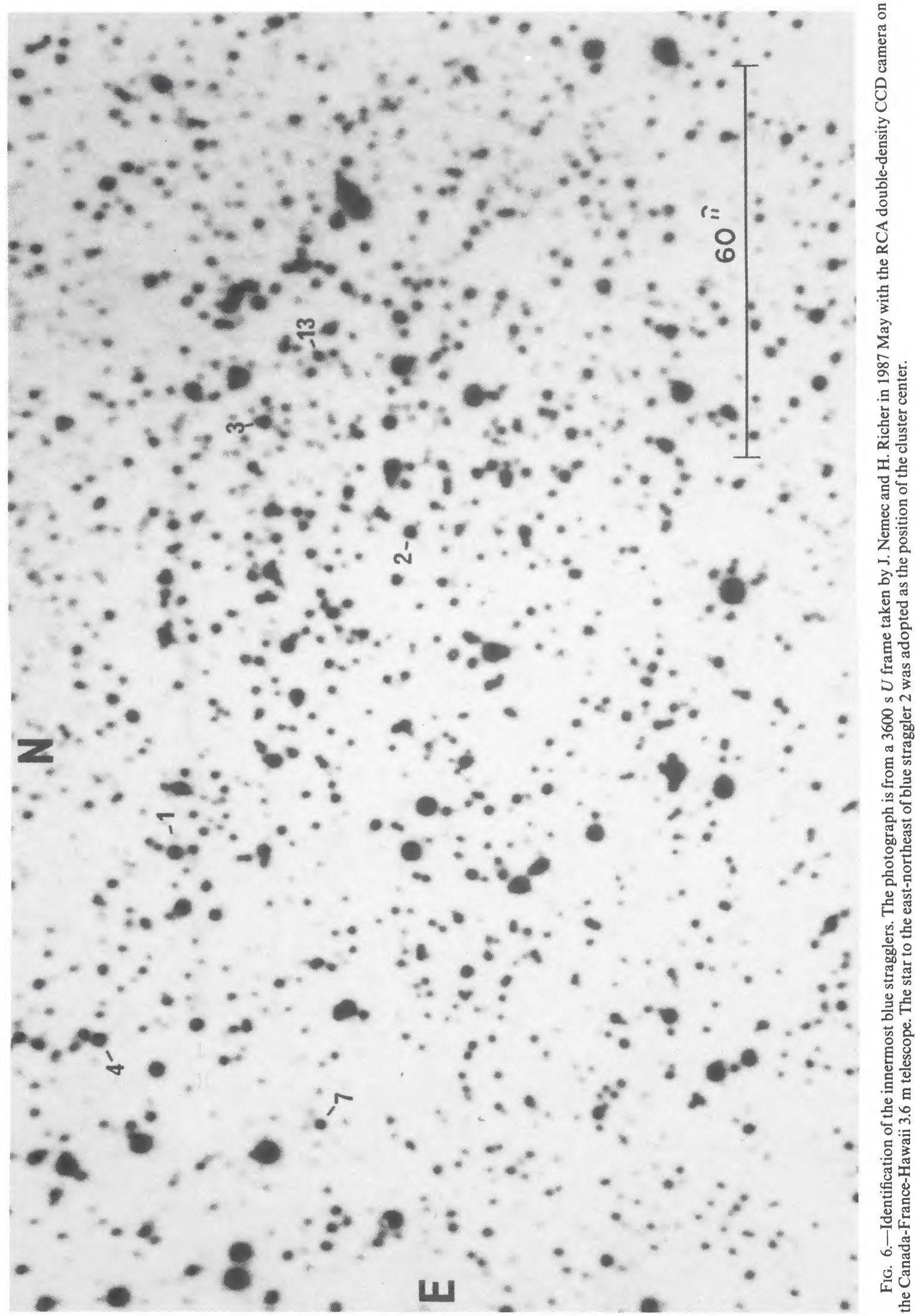




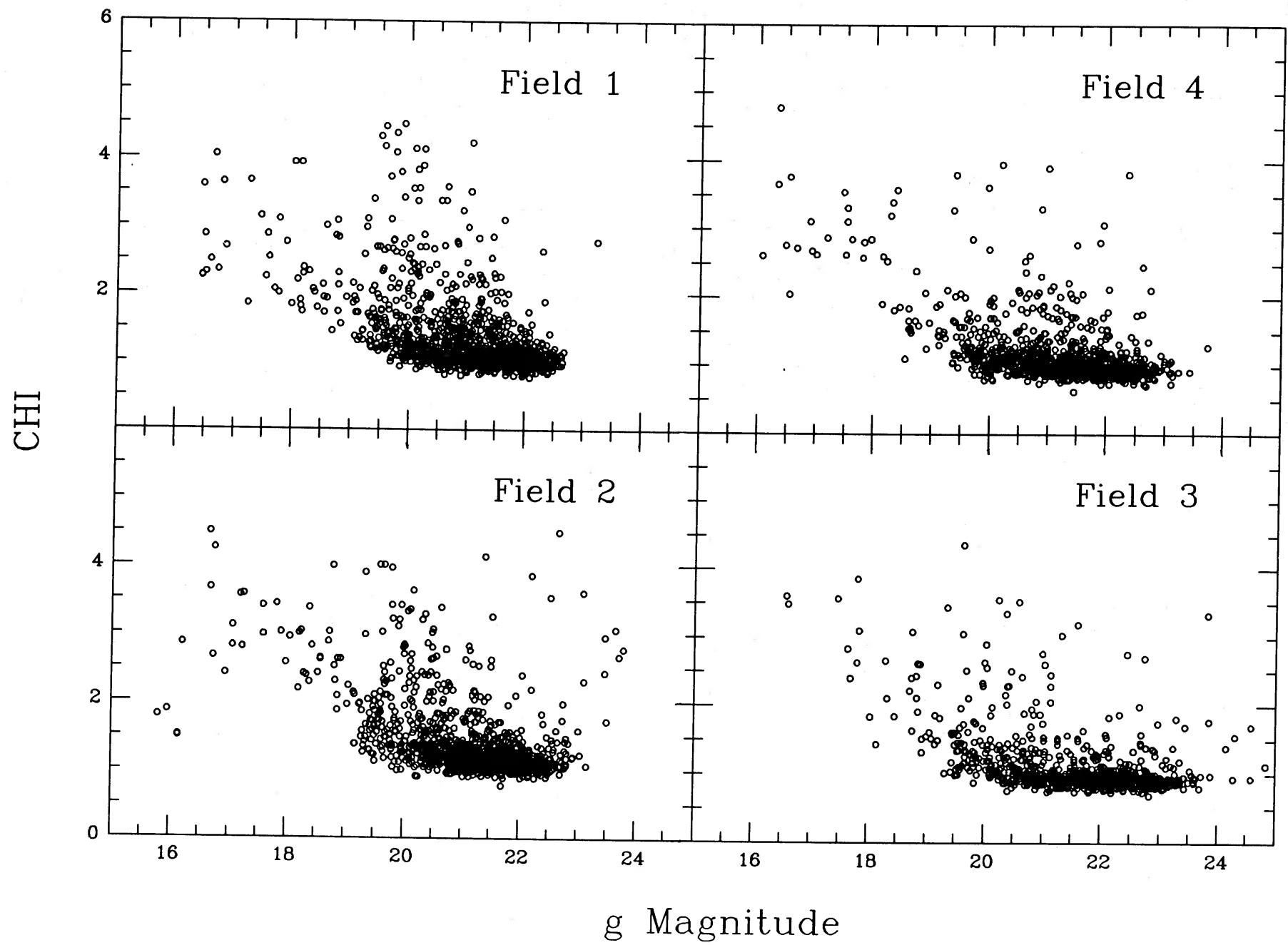
FIG. 8. - CHI values for all the stars measured on the four $r$ frames, plotted as a function of $g$ magnitude. CHI values for the blue stragglers are recorded in
column (9) of Table 6 .

crowded than the center of NGC 5466, and In Paper I it was shown that for an analysis based on similar 4-Shooter frames the blue straggler completeness was $\sim 90 \%$ near the center of NGC 5466.

Contamination of the blue straggler list by field stars should be negligible. The major source of contamination is expected to be field (Galactic halo) main-sequence stars with erroneously blue colors, and cluster subgiants with erroneous colors. The Bahcall and Soneira model of the Galaxy (Ratnatunga and Bahcall 1985) predicts that in the direction of NGC 5053 one expects to see, in $1 \mathrm{arcmin}^{2}$, only 0.063 field stars with colors bluer than $B-V=0.8$ mag (corresponding to $g-r=0.34$ mag) and magnitudes in the range $17 \leq g \leq 19$, and 0.15 field stars in the range $19 \leq g \leq 21$ for the same color interval. Over the $\sim 80 \operatorname{arcmin}^{2}$ field surveyed for blue stragglers, these numbers correspond to five and 12 stars, respectively. However, these are strict upper limits, since the color interval $(B-V) \leq 0.8$ includes field subgiants and main-sequence stars. In the much narrower color range of the NGC 5053 blue stragglers $(-0.5 \leq g-r \leq-0.2)$, one expects (based on the observed ratio of blue stragglers to subgiants) to see at least an order of magnitude fewer stars. Kron's (1980) counts of stars in
SA $57\left(b=85^{\circ}\right)$, and their distribution in the $C$ - $M$ diagram (see Fig. 18 in his paper), shows that even over an $\sim 1080 \mathrm{arcmin}^{2}$ area there are too few field stars in the color range of the blue stragglers to introduce significant contamination of the $C-M$ diagram by field blue horizontal branch stars and RR Lyrae stars. Clearly, the number of blue stragglers identified in the four NGC 5053 CCD fields is far greater than can be accounted for by line-of-sight field stars. It is also not surprising that there are fewer blue stragglers in NGC 5053 than in NGC 5466, given that $M_{V}(\mathrm{NGC} 5466)=-7.1$ compared with $M_{V}(\mathrm{NGC}$ $5053)=-6.1$ (Webbink 1985).

\section{b) Morphology of the Blue Straggler Sequence}

In deep $C$ - $M$ diagrams for M3 (Sandage 1953; Buonanno et al. 1988) and NGC 5466 (Nemec and Harris 1987), the blue straggler sequences are relatively well-defined and narrow, and show a possible "turnover" at high luminosity. Figure 4 shows that this is also the case for NGC 5053. If the "turnoff" toward red colors at high luminosities is real, then more accurate delineation of its structure will be of considerable value for improving our understanding of the evolution of blue stragglers. On the other hand, an apparent turnover in the $C-M$ 


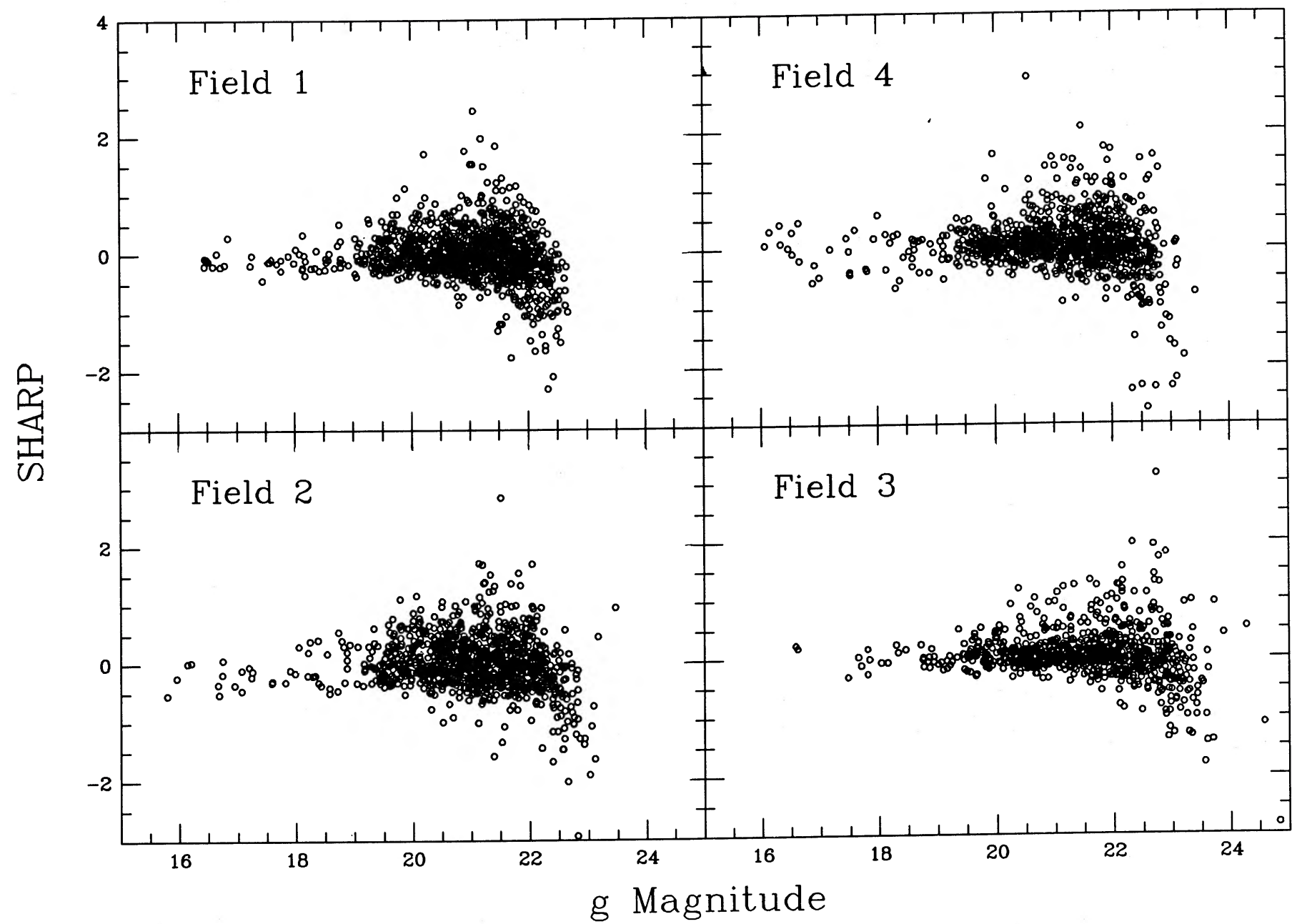

FIG. 9- SHARP values for all the stars measured on the four $r$-frames, plotted as a function of $g$ magnitude. (See Table 6 for the SHARP values for the blue stragglers.)

diagram might also be caused by the presence of variable stars (i.e., dwarf Cepheids) among the blue stragglers, such as those observed among the blue stragglers in $\omega$ Cen (see Da Costa, Norris, and Villumsen 1986). Given that the NGC 5053 blue straggler sequence is defined by a relatively small number of stars, an improved definition of this "turnoff" feature must await further photometry.

\section{c) Radial Distribution of the Blue Stragglers}

In a relaxed stellar system, high-mass stars are expected to have a smaller velocity dispersion and to be situated (on average) closer to the cluster center than low-mass stars. Such mass segregation has recently been observed in the old open cluster M67 (Mathieu and Latham 1986) and in the globular cluster NGC 5466 (Nemec and Harris 1987). In both clusters, the relatively massive blue stragglers were found to be more centrally concentrated than the cluster subgiants. In M67 the blue stragglers follow the projected radial distribution of the spectroscopic binaries in the cluster, and have a mean mass of $\sim 2 M_{\odot}$; and, in NGC 5466, which is not known to contain spectroscopic binary systems, the mean mass of the blue stragglers was shown to be $1.3 \pm 0.3 M_{\odot}$. In both studies, the average mass of the blue stragglers and the main-sequence turnoff stars was determined by fitting the expected radial dis- tributions derived from theoretical multimass King models, to the observed radial distributions.

NGC 5053 is known to be one of the least centrally concentrated globular clusters, with a correspondingly long central relaxation time ( $\sim 5.5 \mathrm{Gyr}$; Peterson and King 1975). Since the relaxation time is about a factor of 3 shorter than the age of the cluster, it follows that NGC 5053 should be in dynamical equilibrium (at least in the central region of the cluster), and there should be equipartition of kinetic energy among the stars of various masses. It also follows that one should be able to determine the mean mass for the blue stragglers using the same method as in Paper I. However, in NGC 5053 one cannot ignore the fact that the high luminosity blue stragglers appear to be more centrally concentrated than the low luminosity blue stragglers (see Fig. 3). This effect is clearly seen in the upper panel of Figure 10, where the apparent $g$ magnitude of the blue stragglers is plotted as a function of projected distance from the cluster center, $R$ (arcsec). A similar trend appears to be present in NGC 5466 but the correlation is much less pronounced (see the lower panel of Fig. 10). Of course, one-half of the difference is due to the bright blue stragglers being bluer than the faint blue stragglers.

To examine the projected radial distributions of the blue stragglers and subgiants, cumulative distribution functions 
NEMEC AND COHEN

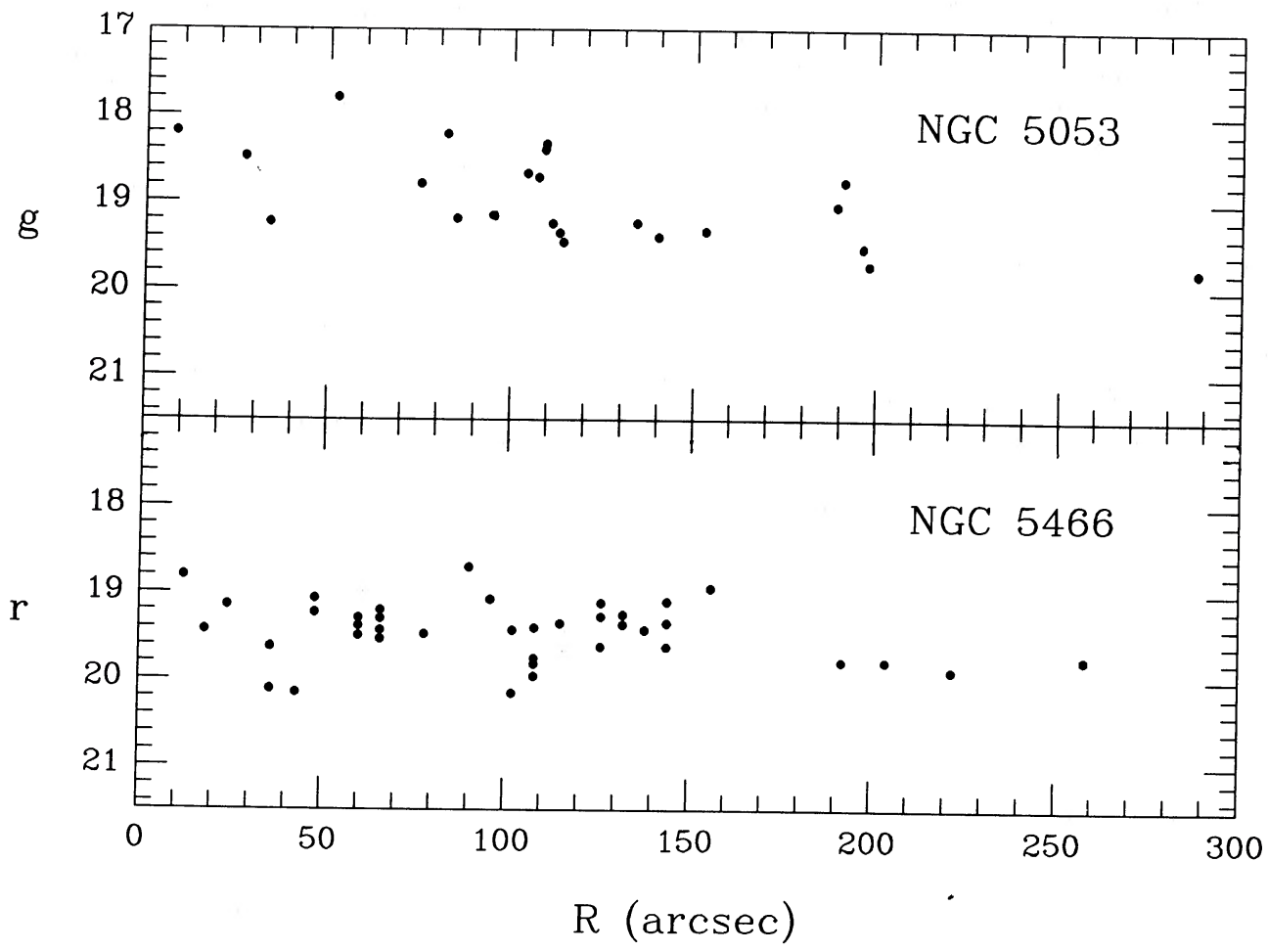

FIG. 10.-The apparent magnitudes of the blue stragglers in NGC 5053 (upper panel) and NGC 5466 (lower panel) are plotted as a function of projected distance from the cluster center, $R$ (arcsec). For NGC 5053 the photometry is from Table 6, and for NGC 5466 the $r$ magnitudes are from Paper I. Blue straggler 20 with
$R=555^{\prime \prime}$ has not been plotted.

(CDFs) with respect to the projected radius, $R$, are plotted in Figure 11 for various stellar sub-populations in NGC 5053. (The CDF is defined to be the fraction of stars with projected radial distance from the cluster center less than or equal to $R$ ).

In the upper left panel of Figure 11 the observed CDF for the 12 brightest blue stragglers (i.e., those in the magnitude range $17.5 \leq g \leq 19.1)$ is compared with the CDF for the 156 subgiants in the same magnitude range. (By comparing stars of similar luminosity, problems arising from differences in the completeness fractions are avoided). To assess the statistical significance of the apparent shift of the blue straggler CDF to the left of the subgiant CDF, a one-sided $t$-test was used to compare the proportion of blue stragglers within the projected distance $R=132$ " (the radius containing half the observed subgiants), to the proportion of subgiants within the same radius. The $t$-statistic formula (given in Paper I) gives a value of $t=1.81$, which corresponds to a probability of $97 \%$. (Because we are interested in testing whether the blue stragglers are more centrally concentrated than the subgiants, the one-sided test is the appropriate test to use). The two CDFs were also compared at all $R$ by performing a one-sided, two-sample Kolmogorov-Smirnov (K-S) test. This test gave a value of 1.29 for the K-S statistic. Assuming that the blue stragglers and the subgiants follow the same projected radial distribution, the probability of obtaining by chance such different CDFs is only $3.6 \%$. (If the very distant blue straggler discovered on the P60 CCD frames, number 20, is excluded from the analysis, the probability drops to only $1.4 \%$ ). We conclude that in NGC 5053 the brightest blue stragglers are significantly more centrally concentrated than the subgiants.

In the upper right panel of Figure 11 the observed CDF for the 12 faintest blue stragglers (i.e., those in the magnitude range $19.1 \leq g \leq 19.8$ ) is compared with the CDF for the 370 subgiants with magnitudes in the same range. In this case the blue straggler CDF appears to be indistinguishable from that of the subgiants. When the CDF for the low-luminosity blue stragglers is compared with that for the corresponding subgiants, the values for the $t$-statistic and the K-S statistic are 1.0 and 0.92 , respectively, and the corresponding probabilities that such values would occur by chance are $50 \%$ and $18 \%$, respectively. Thus, there is no evidence to suggest that the lowluminosity blue stragglers are more centrally concentrated than the subgiants.

The K-S test was also used to test directly whether the 12 high-luminosity blue stragglers are more centrally concentrated than the 12 low-luminosity blue stragglers. The result was a K-S statistic of 1.6 , and a probability of $0.5 \%$ of determining by chance the observed distributions, if the high- and low-luminosity blue stragglers follow the same projected radial distribution. We conclude from this test, and from the indirect tests described above, that the high-luminosity blue stragglers are more centrally concentrated than the low-luminosity blue stragglers. This marks the first time that such mass segregation has been seen within a population of blue straggler stars. (Some evidence for a similar effect in NGC 5466 is seen in Fig 10.)

A comparison of the CDFs of the 156 high-luminosity subgiants and the $\mathbf{3 7 0}$ lower luminosity subgiants is shown in the lower left panel of Figure 11. Here, the K-S test resulted in a statistic of 0.74 , corresponding to a probability of $34 \%$, which implies that there is no evidence to suggest that the high- and low-luminosity subgiants have different projected radial distributions. Assuming that stars with similar radial distribu- 

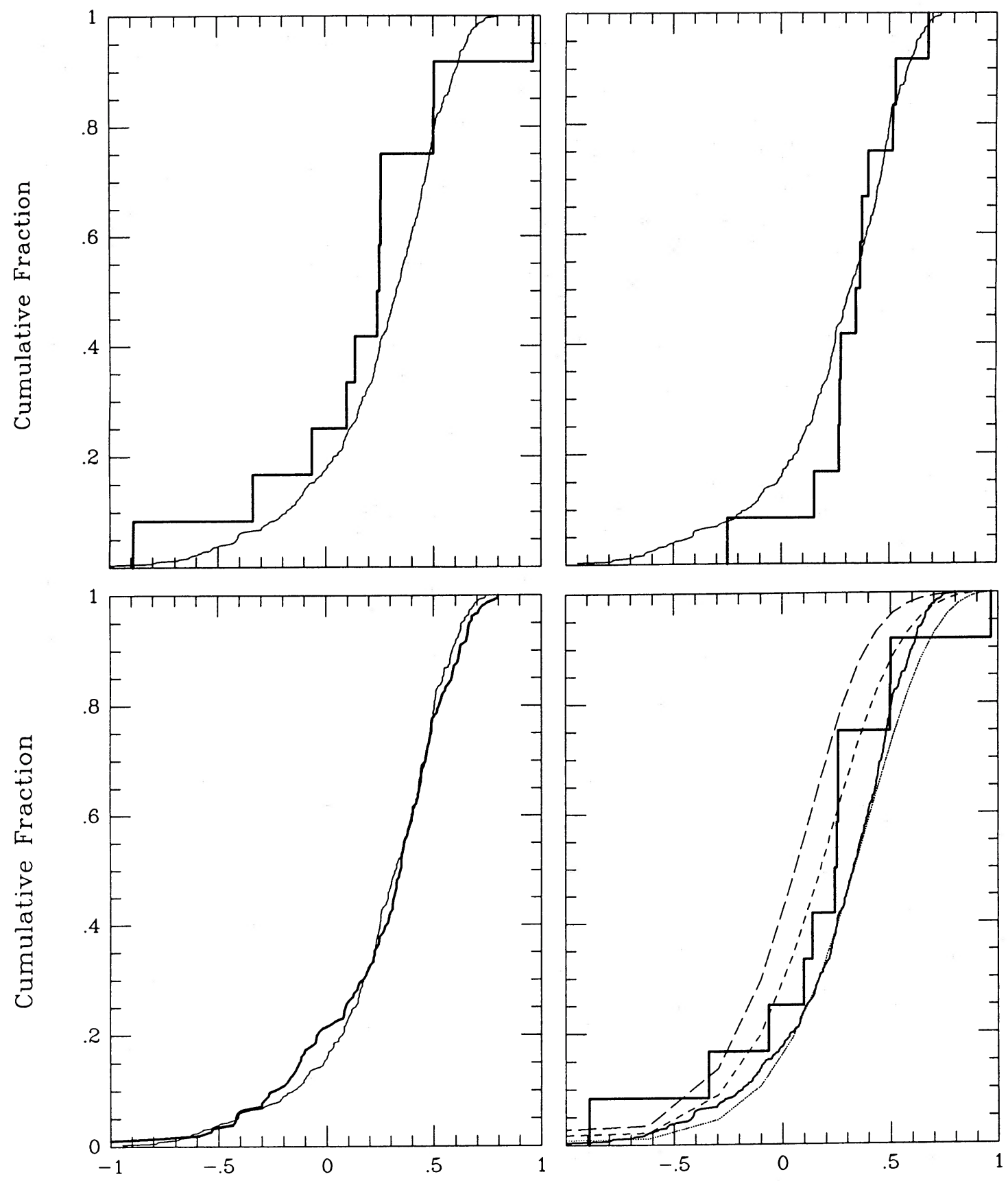

$\log R(\operatorname{arcmin})$

FIG. 11.-(upper left panel) Cumulative distribution function for the 12 brightest blue stragglers, compared with that for the 156 subgiants with magnitudes in the interval $17.5 \leq g \leq 19.1$. A one-sided two-sample K-S test shows that there is only a $3 \%$ chance that the blue stragglers are not more centrally concentrated than the subgiants; (upper right panel) CDF for the 12 low-luminosity blue stragglers, compared with the 370 subgiants with magnitudes in the same interval $19.1 \leq g \leq 19.8$; (lower left panel) Comparing the CDFs of the 156 high-luminosity subgiants and the 370 lower luminosity subgiants. The similarity of the two distributions suggests that the two types of stars have similar masses; (lower right panel) The CDFs for the brightest blue stragglers, compared with the expected distributions for stars of mass $0.8 M_{\odot}$ (the least centrally concentrated), 1.6 $M_{\odot}$ and 2.4 $M_{\odot}$ (the most centrally concentrated). Interpolation suggests that the observed radial distribution for the luminous blue stragglers appears to be most consistent with a mass of $1.3 \pm 0.3 M_{\odot}$. 
tions have similar masses, the subgiants in NGC 5053 probably have masses similar to the mass of a main-sequence turnoff star, $M_{\text {turnoff }} \sim 0.80 M_{\odot}$.

\section{d) Multimass King Models, and the Mean Mass of the Blue Stragglers}

The mean mass of the 12 most luminous blue stragglers in NGC 5053 can be determined by comparing the observed CDFs with expected CDFs computed according to multi-mass King models. In the lower right panel of Figure 11, the CDFs for the 12 brightest blue stragglers (heavy step function), and for the 536 subgiants in the magnitude interval $17.5 \leq g \leq 19.8$ (solid curve), are compared with theoretical CDFs for stars of mass $0.8 M_{\odot}$ (dots), 1.6 $M_{\odot}$ (short dashes), and $2.4 M_{\odot}$ (long dashes). The theoretical CDFs were computed using a multimass King model (see Pryor et al. 1986, and Paper I for references), assuming that the system is in dynamical equilibrium, and that the stars reside in a King (1966) dimensionless central potential $W_{0}=3.45$ (corresponding to a concentration $c=0.75$ ). In an attempt to approximate the true mass distribution in the cluster, NGC 5053 was represented by a model with six mass classes, obeying a mass function of slope $x=1.5$ (Salpeter 1955), i.e., the mass distribution is $\phi(M)=d N / d M=M^{(-1+x)}$, where $d N$ is the number of stars in the mass interval $M$ to $M+d M$. For each mass class, the mean mass per bin $\langle M\rangle=0.25,0.40,0.50,0.60,0.70$, and 0.80 $M_{\odot}$, with corresponding central density fractions $0.25,0.18$, $0.14,0.12,0.11$, and 0.10 , respectively. The CDFs for the 1.6 and $2.4 M_{\odot}$ stars (meant to simulate possible binary blue stragglers) were calculated by adding small fractions of such stars (in separate trial runs of the models), and computing their projected radial distributions.

The CDF in Figure 11 for the 12 brightest blue stragglers would be best fitted by a theoretical CDF intermediate to the 0.8 and $1.6 M_{\odot}$ curves. Interpolation suggests that these stars have a mean mass of $1.3 \pm 0.3 M_{\odot}$. For the subgiants, the $0.8 M_{\odot}$ theoretical CDF provides the best fit to the observations. While the fit is not perfect due to uncertainties in the actual mass function of the cluster, it is adequate for the present purpose of establishing differences in mean masses.

\section{e) Origin of the NGC 5053 Blue Stragglers}

If the NGC 5053 blue stragglers are young, massive, single stars, their mean age can be derived by comparing the Bell and VandenBerg isochrones with the observed blue straggler sequence in the $C$ - $M$ diagram (Fig. 4). In this case, both sets of isochrones $(Y=0.2$ and $Y=0.3)$, with $Z=0.0001$ and $\alpha=1.60$, suggest that the brightest blue stragglers are $\sim 15$ Gyr younger than the majority of the cluster stars. In a system as loosely bound as NGC 5053, it is hard to imagine how a population of relatively massive stars could have formed, with a small velocity dispersion, in a burst of star formation $\sim 3 \mathrm{Gyr}$ ago. We conclude, as was concluded in Paper I for the blue stragglers in NGC 5466, that it is unlikely that the blue stragglers are young and single.

On the other hand, the two leading hypotheses if the blue stragglers are old are as follows: (1) they are relatively massive single stars with main-sequence lifetimes extended by the partial mixing of unprocessed stellar material into nuclear burning regions of the star (Wheeler 1979; Saio and Wheeler 1980; Da Costa and Demarque 1982; VandenBerg and Smith 1988); or, (2) they are mass-transfer binary systems (Hoyle 1964; McCrea 1964; RMS). The mixing hypothesis has the drawback that it is difficult to verify observationally and can only be proved indirectly by ruling out the binary hypothesis. On the other hand, the binary hypothesis makes concrete predictions that can be tested observationally: (1) the most massive blue stragglers are not expected to be more massive than twice $M_{\text {turnoff }}$; (2) the absolute magnitude of the most luminous blue straggler is not expected to be more than 2.5 mag brighter than the main-sequence turnoff stars; and (3) if anomalous Cepheids represent an evolutionary stage through which blue stragglers more massive than $1.3 M_{\odot}$ will pass (RMS; Carney and Seitzer 1986), then the ratio of blue stragglers to anomalous Cepheids, $N_{\mathrm{BS}} / N_{\mathrm{AC}}$, is expected to be $\sim 1-10$ (RMS).

The present study gives results that are consistent with predictions (1) and (2) above: first, the mean mass of the brightest blue stragglers in NGC 5053 does not exceed $2 M_{\text {turnoff }}$; and second, the difference between the luminosity of the most luminous blue straggler, and the luminosity of the mainsequence turnoff stars, does not exceed $2.5 \mathrm{mag}$. Prediction (3) cannot be tested directly because NGC 5053 contains no anomalous Cepheids. However, it is of related interest that Nemec, Wehlau, and Oliveira (1988) have recently found that $N_{\text {BS }} / N_{\text {AC }}$ in the Ursa Minor dwarf galaxy is only $\sim 10$ (and not $\lesssim 100$ as had been computed previously), which agrees well with prediction (3). Furthermore, Nemec, Wehlau, and Oliveira also show that the maximum mass of the most luminous anomalous Cepheid in the Draco dwarf galaxy is consistent with the prediction of the binary hypothesis.

Given that the "mass transfer in a close binary system" hypothesis continues to meet all observational challenges and is the only hypothesis that readily lends itself to observational testing, it is our opinion that it remains the leading candidate for explaining blue stragglers. If blue stragglers are binary systems, the most important unanswered question would seem to concern the separation of the component stars. The reader is referred to Nemec and Harris (1987) for a more detailed discussion of blue stragglers.

\section{SUMMARY}

The two main conclusions of this study are that NGC 5053 has an age of $18 \pm 3 \mathrm{Gyr}$, according to the best fit of the Bell and VandenBerg (1987) theoretical isochrones to the observed main-sequence turnoff and subgiant branch regions of the $C-M$ diagram (assuming $Y=0.25, Z=0.0001, \alpha=1.6$ ); and that the cluster contains a population of at least 24 blue stragglers. The projected radial distribution of the 12 most luminous blue stragglers is found to be significantly more centrally concentrated than that of the cluster subgiant and upper mainsequence stars. We also find that the 12 high-luminosity blue stragglers tend to be located nearer to the cluster center than the 12 lower luminosity blue stragglers. Comparison with multimass King models suggests that the most luminous blue stragglers have an average mass, $\left\langle M_{\mathrm{bl.str}}\right\rangle=1.3 \pm 0.3 M_{\odot}$, which is comparable to but just under twice the mean mass of the cluster subgiant and main-sequence turnoff stars. A mainsequence luminosity function has been plotted for the subgiants and main-sequence stars in NGC 5053, and it shows no sign of "turning over" for stars brighter than $M_{g} \sim 5 \mathrm{mag}$.

In the future, it will be desirable to determine the fraction of NGC 5053 blue stragglers that are dwarf Cepheids. ${ }^{4}$ It will also

\footnotetext{
${ }^{4}$ Nemec et al. (1988) have recently identified blue stragglers 7, 11, 13 and 14 as certain SX Phoenicis variables.
} 
be valuable to measure accurate radial velocities for the cluster red giants and blue stragglers and to derive their average masses using the virial theorem (see Bahcall and Tremain 1981). A comparison of the virial theorem mass with the mean mass derived here for the high-luminosity blue stragglers would be of considerable interest. According to the multimass King models, the predicted velocity dispersion for $0.8 \mathrm{M}_{\odot}$ stars is $1.1 \mathrm{~km} \mathrm{~s}^{-1}$, while the velocity dispersions for $1.6 M_{\odot}$ and $2.4 M_{\odot}$ stars are only $0.8 \mathrm{~km} \mathrm{~s}^{-1}$ and $0.7 \mathrm{~km} \mathrm{~s}^{-1}$, respectively. A thorough survey for blue stragglers in many globular clusters, to map the morphology of blue straggler sequences in $C-M$ diagrams (in particular, the apparent "turnoff" at high luminosities), for clusters with a range of metal abundances and central concentrations, would permit an improved picture of the blue stragglers to be drawn. The relative contribution of the total blue straggler light to the total integrated light of the cluster should also be made.

We thank Don VandenBerg and Roger Bell for kindly pro- viding the theoretical isochrones in advance of publication, Carlton Pryor for the use of his programs from which the multimass King models were computed, Harvey Richer and Greg Fahlman for useful discussions and for permission to reproduce the deep CFHT CCD frame, and Alain Porter for providing the information on the 4-Shooter readout noise and photons $\mathrm{ADU}^{-1}$. We also thank Amanda Nemec for a careful reading of the paper, Peter Stetson for valuable discussions concerning the photometry, and the anonymous referee for some important suggestions. David Duncan assisted in making some of the photographic prints. J. G. C. is grateful to Caltech and to the Caltech Recycling Center for financial support. J. M. N. gratefully acknowledges a Canada Council Killam Postdoctoral Fellowship (1985-1987), and the Natural Sciences and Engineering Research Council of Canada for a Postdoctoral Fellowship at Caltech, and for a University Research Fellowship at the University of British Columbia.
Aarseth, S. J., and Lecar, M. 1975, Ann. Rev. Astr. Ap., 13, 1.

Baade, W. 1927, Astr. Nach., Vol. 232, No. 5555.

Bahcall, J. N., and Tremaine, S. 1981, Ap. J., 244, 805.

Bell, R. A., and Gustafsson, B. 1983, M.N.R.A.S., 204, 249.

Bell, R. A., and VandenBerg, D. 1987, Ap. J. Suppl., 63, 335.

Boesgaard, A. M., and Steigman, G. 1985, Ann. Rev. Astr. Ap., 23, 319

Buonanno, R., Buzzoni, A., Corsi, C. E., Fusi Pecci, F., and Sandage, A. R.

1988 , in IAU Symposium 126, The Harlow-Shapley Symposium on Globular

Cluster Systems in Galaxies, ed. J. E. Grindlay and A. G. Davis Philip

(Dordrecht: Reidel), p. 621.

Burstein, D., and Heiles, C. 1982, A.J., 87, 1165.

Carney, B., and Seitzer, P. 1986, A.J.,92, 23.

Chernoff, D. F., and Shapiro, S. L. 1987, Ap. J., 322, 113.

Cohen, J. G. 1985, A.J., 90, 2254.

Cuffey, J. 1943, Ap. J., 98, 49.

Da Costa, G. S., and Demarque, P. 1982, Ap. J., 259, 193.

Da Costa, G. S., Norris, J., and Villumsen, J. V. 1986, Ap. J., 308, 743.

Fahlman, G., Richer, H., and Nemec, J. 1989, in preparation.

Gunn, J. E., et al. 1984, Bull. A.A.S., 16, 447.

Heasley, J., and Christian, C. 1986, Ap. J., 307, 738.

Hills, J. G., and Day, C. A. 1976, Ap. Letters, $17,87$.

Hoyle, F. 1964, Roy. Obs. Bull., 82, 91.

Iben, I., Jr., and Rood, R. T. 1970, Ap. J., 161, 587.

Kent, S. M. 1985, Pub. A.S.P., 97, 165.

King, I. 1962, A.J., 67, 471.

King, 1966, A.J., 71, 64

Kron, R. G. 1980, Ap. J. Suppl., 43, 305.

Kunth, E. D., and Sargent, W. L. W. 1983, Ap. J., 273, 81.

Mannino, G. 1963, Pub. Obs. Bologna, Vol. 8, No. 12.

Mathieu, R. D., and Latham, D. W. 1986, A.J., 92, 1364.

McCrea, W. H. 1964, M.N.R.A.S., 128, 147.

McClure, R. D. 1984, Ap. J. (Letters), 280, L31.

McClure, R. D., Fletcher, J. M., and Nemec, J. M. 1980, Ap. J. (Letters), 238 L35.

McClure, R. D., Hesser, J. E., Stetson, P. B., and Stryker, L. L. 1985, Pub. A.S.P., 97,665 .

McClure, R. D., and Norris, J. 1977, Ap. J.(Letters), 216, L101.

\section{REFERENCES}

McClure, R. D., et al. 1986, Ap. J. (Letters), 307, L49.

Nemec, J. M. 1989, in preparation.

Nemec, J. M., and Harris, H. C. 1987, Ap. J., 316, 172 (Paper I)

Nemec, J. M., Mateo, M., Burke, M., Harris, H. C., Richer, H., and Fahlman, G. 1988 , in preparation.

Nemec, J. M., Wehlau, A., and Mendes de Oliveira, C. 1988, A.J., 96, 528.

Norris, J., and Zinn, R. 1975, Ap. J., 202, 335

Peterson, C. J., and King, I. R. 1975, A.J., 80, 427.

Peterson, R., Carney, B., and Latham, D. 1984, Ap. J., 279, 237.

Pryor, K., McClure, R. D., Fletcher, J. M., Hartwick, F. D. A., and Kormendy, J. 1986, A.J., 91, 546.

Ratnatunga, K. U., and Bahcall, J. N. 1985, Ap. J. Suppl., 59, 63.

Renzini, A., and Fusi Pecci, F. 1988, Ann. Rev. Astr. Ap., 26, in press.

Renzini, A., Mengel, J. G., and Sweigart, A. V. 1977, Astr. Ap., 56, 369 (RMS).

Rosino, L. 1949, Pub. Oss. Bologna, 5, 10.

Saio, H., and Wheeler, J. C. 1980, Ap. J., 242, 1176.

Salpeter, E. E. 1955, Ap. J., 121, 161.

Sandage, A. R. 1953, A.J., 58, 61. 1970, Ap.J., 162,841. 1983, A.J., 88, 1159 .

Sandage, A. R., Katem, B., and Johnson, H. 1977, A.J., 82, 389 (SKJ).

Sandage, A. R., and Smith, L. L. 1966, Ap. J., 144, 884.

Sawyer, H. 1946, Pub. David Dunlap Obs., 1, 357.

Sawyer Hogg, H. 1973, Pub. David Dunlap Obs., Vol. 3, No. 6.

Searle, L., and Zinn, R. 1978, Ap. J., 225, 357.

Shawl, S. J., and White, R. E. 1986, A.J.91, 312.

Smith, G. H., McClure, R. D., Stetson, P. B., Hesser, J. E., and Bell, R. A. 1984 A.J., 91, 842.

Stetson, P. B. 1986, DAOPHOT User's Manual.

Stetson, P. B. 1986, DAOPHOT User

Suntzeff, N. B., Kraft, R. P., and Kinman, T. D. 1988, A.J., 95, 91.

Thuan, T. X., and Gunn, J. E. 1976, Pub. A.S.P., 88, 543.

VandenBerg, D. A., and Smith, G. H. 1988, Pub. A.S.P., 100, 314.

Webbink, R. F. 1985, in IAU Symposium 113, Dynamics of Star Clusters, ed.

J. Goodman and P. Hut (Dordrecht: Reidel), p. 541.

Wheeler, J. C. 1979, Ap. J., 234, 569.

Zinn, R., and Searle, L. 1976, Ap. J., 209, 734.

Judith G. CoHEN : Department of Astronomy, California Institute of Technology, Pasadena, CA 91125

JAMES M. NEMEC: Department of Astronomy, University of British Columbia, Vancouver, B.C., V6T 1W5, Canada 